\title{
Modeling the early steps of cytoplasmic trafficking in viral infection and gene delivery
}

\author{
C. Amoruso, T. Lagache D. Holcman, \\ Department of Applied Mathematics and Computational Biology, IBENS \\ Ecole Normale Supérieure, 46 rue d'Ulm 75005 Paris, France
}

September 2, 2021

\begin{abstract}
Gene delivery of nucleic acid to the cell nucleus is a fundamental step in gene therapy. In this review of modeling drug and gene delivery, we focus on the particular stage of plasmid DNA or virus cytoplasmic trafficking. A challenging problem is to quantify the success of this limiting stage. We present some models and simulations of plasmid trafficking and of the limiting phase of DNA-polycation escape from an endosome and discuss virus cytoplasmic trafficking. The models can be used to assess the success of viral escape from endosomes, to quantify the early step of viral-cell infection, and to propose new simulation tools for designing new hybrid-viruses as synthetic vectors.
\end{abstract}

Keywords: Mathematical Modeling, Gene Delivery, Stochastic Processes, Cytoplasmic Trafficking, modeling early steps of infection, Endosomal Escape, Narrow escape.

\section{Introduction}

Drug delivery is a multi-step process of delivering a cocktail of molecules through different tissue levels to a specific location in the body, which is usually a difficult task. For example, the Brain Blood Barrier is a physical barrier that drastically limits the access of molecules to the central nervous system, preventing efficient drug delivery. The possibility of injecting to a cell a piece of DNA that can reach the nucleus, in order to synthesize a given protein, is a very seducing idea, which has driven gene therapy and genetic crop modification. Gene therapy has been tried in the quest to cure diseases caused by single-gene defects, such as cancer and hereditary diseases linked to a genetic defect [1].

Non-viral methods for gene delivery use a variety of tools such as polymeric gene carriers, microinjection, gene gun, hydrostatic pressure, electroporation, continuous infusion, and many others [1]. Gene delivery can be used, in principle, for fighting major diseases, ranging 
from viral infection to epilepsy. Although viruses are great predators, they can serve as vectors of drugs. For example, the Adeno-Associated Virus of type 2, (parvoviruses [2]) can carry gene coding for the neuropeptide $\mathrm{Y}$, which is transduced in some areas of the hippocampus, where it is injected. The peptide is then released from neurons during high brain stimulations and reduces glutamate release, thus preventing the propagation of a high firing rate in neighboring neurons [3]. This therapy has the potential to reduce epileptic form activity.

In this review, we discuss recent models of intracellular delivery and viral trafficking. Mathematical and physical models are constructed for the purpose of predicting and quantifying infectivity and the success of gene delivery. The models give rise to rational Brownian dynamics simulations for the study of sensitivity to parameters and, eventually, for testing the increase or the drop in infectivity, by using simultaneously a combination of various drugs. The modeling approach can be used for the optimization of the delivery in a highdimensional parameter space. In the first part of the review, we present several approaches to the study of some limiting steps of gene delivery. A major limiting step remains the success of plasmid escape from an endosomal compartment. In the second part, we present models of viral entry, the endosomal step and trafficking in the cytoplasm. These models can be used to predict the efficacy of new synthetic vectors, based on hybrid viruses.

\section{Mechanisms of gene delivery.}

\section{Introduction: Two types of gene vectors}

Gene therapy is a new therapeutic strategy that offers the promise of treating diseases through insertion, alteration, or removal of genes within an individual's cells and biological tissues. In general, any drug molecule must reach its intended site of action to exert its effect and to avoid non specific potentially dangerous interactions. A daunting hurdle of drug delivery is the large amount of charged molecules such as DNA that need to reach the cell nucleus. Indeed, the DNA molecule must initially pass through the cell membrane, traffic inside the cell cytoplasm to finally enter the nuclear pore in a form suitable for transcription to start. To overcome efficiently these barriers, the genetic material is usually associated to a viral or synthetic vector (see figure 1). Viruses have developed evolutionary tools to enter cells and get transported towards the different compartments such as the nucleus and finally to reproduce using the protein synthesis machinery of the host cell (see figure 1-b). However their use presents several limitations: viral vectors are not safe and have been implicated in death during clinical trials [4], they can trigger an immune response and thus cannot be administrated repeatedly. Finally, the size of the transferred genome is limited by the nucleocapsid architecture. To overcome the difficulties imposed by the use of viral particles as vectors, synthetic vectors such as lipids, cationic polymers, peptides or combinations thereof, have been developed in parallel. In particular, the most common used vectors for nonviral gene delivery are cationic lipids, first introduced in 1987 [5] and later on cationic polymers [6], followed by improved [7] polycations such as the polyethylenimine (PEI) for transfection experiments. Cationic lipids are macromolecules consisting of an 
a)

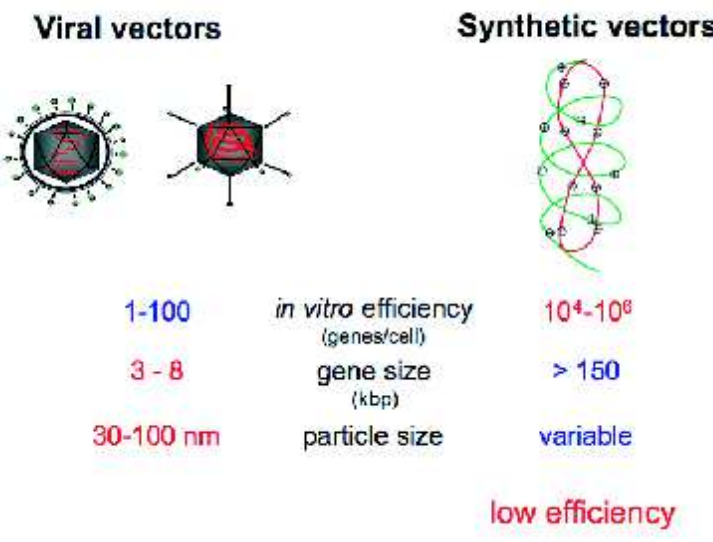

b)

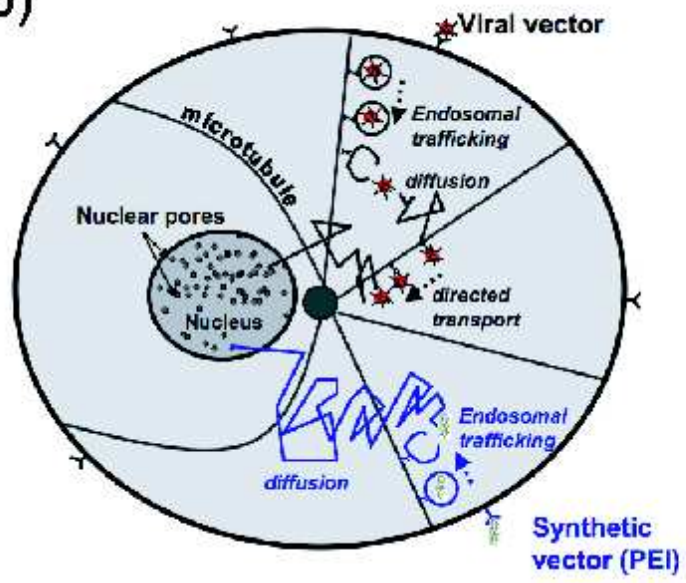

Figure 1: Viral and synthetic vectors are used to transfer genes in the nucleus. (a) characteristics of viral and synthetic vectors. While synthetic vector polycations are preferred to viruses, they are safer and the size of the genes is not limited by any capsid architecture, but their efficiency to escape endosomes and traffic through the risky cytoplasm is still very low. (b) schematic description of early step of infection for viral and synthetic vectors. Synthetic vectors are not assisted by active transport during their cytoplasmic trafficking.

aqueous core enclosed in a spherical phospholipid layer with positively charged head groups, while polycations are positively charged polymers which can have different structures (linear or branched). Both molecules are able to condense and neutralize DNA molecules through electrostatic interactions between their positive charges and the negatively charged DNA phosphate groups. In both cases, polycations protect DNA from undesirable degradation and facilitate entry into cells.

Despite the high degree of reproducibility, the ease of modification, the lack of toxicity and the ability to deliver large pieces of DNA, polycations and liposomes are still much less efficient than their viral counterpart due to low cellular uptake and endosomal escape. A new promising strategy is the use of cationic liposomes incorporating fusogenic peptides from viral glycoproteins, which are supposed to enhance endosomal escape by mimicking the mechanism of fusion of viral envelopes with host cell endosomal membrane [8, 9].

Unraveling the molecular mechanisms that underly the cellular behavior of both viruses and synthetic vectors is now needed to develop and optimize efficient hybrid vectors. In particular, the endosomal escape remains a major barrier in gene delivery and prompt release from an endosomal compartment presumably constitutes one of the critical steps in determining the efficiency of transfection. In addition, the cytosolic motion of large DNA molecules is limited by physical and chemical barriers of the crowded cytoplasm [10, 11]: whereas molecules smaller than $500 \mathrm{kDa}$ can diffuse, larger cargos such as viruses or synthetic gene vectors, require an active transport system [12] such as the microtubules (MTs). Consequently, to understand how molecular components of gene vectors can affect quantitatively their ability to pass through these two main limiting barriers of gene expression, we will present a review on our recent biophysical models for both viral and synthetic vectors. Indeed, while modeling the endosomal escape of viruses will help to understand the molecular 
mechanisms underlying their reliable escape at a given $\mathrm{pH}$, a model of the free cytoplasmic step will allow to analyze the active transport along MTs, which increases the probability that a viral particle reaches a nuclear pore in comparison with a Brownian synthetic gene vector.

\section{The cellular uptake pathways}

Since the phospholipid bilayer of the plasma membrane has a hydrophobic exterior and a hydrophobic interior, any polar molecules, including DNAs and proteins, are unable to freely pass through cell membranes. To circumvent this barrier, an approach consists in transferring naked nucleic acids by inducing a membrane destabilization. An externally applied electrical field can temporarily disrupt areas of the membrane allowing polar molecules to pass, then the membrane can reseal quickly and leave the cell intact [13, 14, 15]. This method, commonly known as electropermeabilization or electroporation, provides versatility and efficiency, but it suffers from non specific transport across the membrane which can lead to ion imbalance and thus improper cell functions. It can also lead to irreversible cell damage if the electrical field is too strong. Microinjection also permits rapid delivery of genes to the cytosol or the nucleus by using a glass micropipette to inject genetic material into cells.

Permeabilization is another used technique of delivery in which pore-forming agents which have the ability to fuse with the membrane are used to form large apertures in the cell membrane. However these techniques are highly invasive and cannot be used for in vivo gene delivery. A different strategy to overcome the initial cell membrane barrier is by associating genetic molecules with viral or synthetic vectors such as lipids, cationic polymers, peptides or combinations thereof, which are internalized through the endocytic pathway. Endocytosis is the process by which cells absorb molecules by engulfing and enclosing them into vesicles. There are several different endocytic pathways:

1. Clathrin-Mediated Endocytosis Clathrin-Mediated Endocytosis (CME) is a process by which cells internalize molecules through the strong binding of a ligand to a specific cell surface receptor. This process results in the clustering of the ligand-complexes in coated pits which are formed by cytosolic proteins, the main unit being clathrin. The coated pits then invaginate the plasma membrane to form clathrin-coated vesicles.

2. Caveolae-Mediated Endocytosis Caveolae are small (approx. $50 \mathrm{~nm}$ in diameter) pits in the membrane that resemble the shape of a cave which can mediate uptake of extracellular molecules through complex signaling.

3. Macropinocytosis A mechanism of endocytosis in which large droplets of fluid are trapped underneath extensions (ruffles) of the cell surface.

4. Phagocytosis is the process by which cells bind and internalize large $(>0.5 \mu)$ pathogens such as bacteria or micro-organisms. 


\section{Endosomal escape}

Once a gene vector (synthetic or viral) enters an endosome, it has to escape into the cytoplasm before being degraded in lysosomes. Although the exact pathways leading to endosomal escape are not fully elucidated, they are limiting steps in gene delivery. Most viruses possess efficient endosomolytic proteins allowing them to disrupt the endosomal membrane, such as the VP1 penetration protein of the adeno-associated virus (AAV) [16] or the influenza hemagglutinin (HA), [17]. In addition, the biophysical mechanism leading to endosomal membrane destabilization and concomitant plasmids release for synthetic vectors is still poorly understood. However, in both cases, acidification of the endosome is needed to trigger endosomal escape. For viruses protons or low $\mathrm{pH}$ activated proteases bind viral endosomolytic proteins, triggering their conformational change into a fusogenic state [17, 16]. Polyplexes (DNA/cationic polymer) use a different strategy to escape endosomal compartments, based on the so-called proton sponge mechanism. Indeed some polycations have a high buffering capacity in a wide range of $\mathrm{pH}$ thus preventing the DNA from degradation and inducing an influx of chloride $\mathrm{Cl}^{-}$ions to maintain electrical neutrality. The osmotic pressure due to the influx of $\mathrm{Cl}^{-}$ions causes endosome swelling and eventually membrane disruption.

\section{Cytoplasmic trafficking}

Following the endosomal escape, viral and synthetic gene vectors have to travel through the crowded cytoplasm to reach the nucleus and deliver their genetic material through the nuclear pores. While the cytoplasmic movement of viral particles towards the nucleus is facilitated by the microtubular network and viral proteins, very little is known about the fate of non-viral DNA vectors in the cytoplasm. However, trapping of large DNA particles (>500 kDa) in the crowded cytoplasm drastically hinders their cytoplasmic diffusion [10, 11] and subsequently diminishes the transfection rate of synthetic gene vectors. In addition, one of the critical barriers in polyplex-mediated gene delivery is the timely unpacking process of the complexes within the target cell to liberate the DNA for efficient gene transfer [18]. A desirable property of a gene carrier is that it strongly binds to DNA to form more compact structures that can provide better protection of genetic material against nucleases and efficient transportation through the cytoplasm. However stronger polycation-DNA interactions produce a counterproductive effect on the timely release of DNA for transcription. Therefore an ideal vector would be capable of binding strongly to the DNA during the early stages of intracellular transport and to release the DNA right before nuclear entry.

\section{Nuclear delivery}

Expression of therapeutic proteins in gene therapy requires that the transfection agent, a virus or a synthetic vector, delivers its cargo through the nuclear envelope (NE) into the cell nucleus. Later on, the genetic material will be involved in the cellular transcriptional machinery. Some viruses such as AAV are able to transiently disrupt the NE [19], delivering their intact capsid for disassembly inside the nucleus. However, most viruses translocate 
inside the nucleus through nuclear pore complexes (NPCs) by using nuclear localization sequences (NLSs). This facilitated nuclear import can accommodate the transport of molecules with diameters of up to $39 \mathrm{~nm}$ [20, 21]. Thus, small viruses such as hepatitis B virus [22] or baculoviruses [23] can cross the NPC and disassemble inside the nucleus. In contrast, larger viruses such as HIV [24] or influenza [25] uncoat inside the cytoplasm, or near a NPC, for the adenovirus [26]. The released components contain NLSs and are thereby able to cross the NPC. Viral derived NLS peptides associated to polycations have also appeared to enhance the nuclear delivery of genes [27].

Recent biophysical models have been proposed to quantify the molecular mechanisms of viral facilitated transport through the NPC [28, 29, 30, 31], however the exact import mechanisms still remain unclear. Interestingly, it has been found that pressure due to DNA condensation in double-stranded DNA viruses, such as the herpes virus, is as high as 50 atmospheres, allowing a direct injection of the genome inside the nucleus through the NPC [32].

\section{Modeling the endosomal pathway in Gene Delivery with cationic lipids and polycations.}

The relative inefficiency of transfection using polycations, compared to the use of viral vectors remains the largest barrier to synthetic vector development and applications. Elucidating how non-viral vectors behave at the intracellular level is therefore a necessary step for synthetic vector improvement and optimization [33. Biophysical modeling of polyelectolytes in confined domains, their interaction with biological membranes and escape from endosomes would allow us to design new efficient vectors. Computational models have accounted for endosomal escape using kinetic equations [34], where parameters describing multiples steps of gene delivery pathways are specified from experiments, numerical fits or through statistical mechanics analysis [35]. However, it is difficult to extend these methods to identify the biophysical mechanisms underlying DNA endosomal escape.

\section{Two biophysical scenarios to study the endosomal release of DNA}

Cationic polymers, such as PEI, condense DNA into nano-sized polymer/DNA complexes (polyplexes) by a self-assembling process due to electrostatic interactions of the positively charged polymer with the negatively charged DNA. Polyplexes with positive surface charges are formed when the number of positive charges of the polymer exceed that of the negative DNA charges. When a polyplex encounters the cell surface, it interacts with the negatively-charged cellular membrane and can be taken up into the cells via endocytosis. In the intracellular environment the polyplexes are located in endosomes that become acidified. In this case, DNA is prone to degradation by lysosomal enzymes. In order to transfer DNA cargo to the nucleus, polyplexes must escape from endosomes. This transmembrane mechanism or endosomolytic process remains unclear and it is believed that high gene transfection 

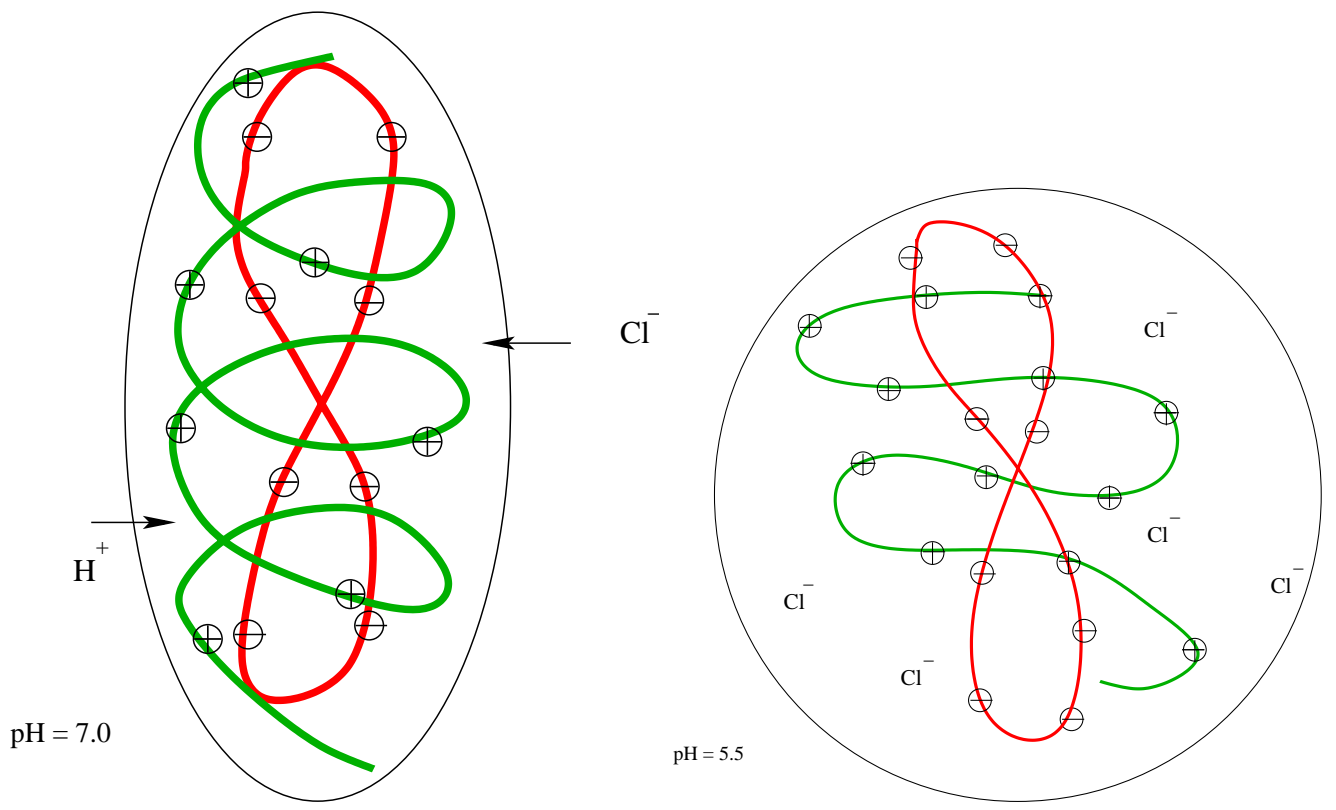

Figure 2: The proton sponge hypothesis: protons $\mathrm{H}^{+}$and chloride ions $\mathrm{Cl}^{-}$enter into the endosome. The increased $\mathrm{Cl}^{-}$concentration in the endosome with respect to the cytosol causes an osmotic pressure on the endosomal membrane which can induce its disruption. Swelling mechanism: Protons bind to the polycation thus increasing its charge density and stiffness due to higher Coulomb repulsion between monomers. In the figure, the darker chain represents the DNA while the lighter one represents the polycation molecule.

efficiency observed with PEI is due to its ability to avoid endosome acidification and to promote complexe's release through a mechanism called "proton sponge". This mechanism can be summarized as follow (2): the PEI contains amine groups, which can be protonated at low $\mathrm{pH}$. Thus due to an influx of chloride ions $\mathrm{Cl}^{-}$to maintain the overall electroneutrality, and water molecules, the endosome starts swelling leading to an increased osmotic pressure, a destabilization of the endosome and ultimately disruption. It has not been proved yet whether the stress produced by the proton sponge effect and by the swelling are the only mechanisms responsible for the endosomal membrane disruption. However, recent experimental evidences argue in favor of this escape mechanism [36]. There are other possible mechanisms responsible for endosomal disruption such as swelling of the endosome due to internal charge repulsion triggered by an increased of ion concentration in the endosome. This effect implies a direct physical interaction between the DNA-PEI complex and the endosomal membrane. The PEI-DNA complex can escape an endosome through a membrane hole, probably due to direct interaction of polymers with membrane [37]. For example, the interaction between the cationic polymer PEI and cellular components [38, 39] lead to Gramnegative bacteria membrane disruption by free PEI, [40]. Furthermore, PEI also causes a liposomal membrane permeability to increase [41]. Thus, PEI is capable of destabilizing lysosomes and this suggests that it enables the DNA conjugated to the PEI to escape into the cytoplasm. Finally, a wide variety of nanoparticles, including different cationic polymers can physically disrupt lipid membranes by forming nanoscale hole and membrane thinning [42]. Cationic polymers contribute to expand existing defects or can directly induce small holes. 
Today, most common theories of pore formation are derived from the classical nucleation theory [43], which unfortunately does not predict stable pores of finite size. However long-lived pores that remained open for several seconds have been observed [44, 45, 46, 47, 48, Several theoretical models have succeeded to show that after pores nucleation, their enlargement is expected to relax the surface tension, leading to the formation of a stable or long-lived metastable pore [49, 50, 51, 52, 53, 54].

For a planar membrane, increasing the lipid density occurs concurrently to pore dilation, that reduces mechanical tensions. After a vesicle opens, its internal content escapes, reducing the osmotic pressure and the associated surface (Laplace) tension. Although membrane fluctuation was recently modeled [55, 56, 57], there is still no consistent theory of pore formation and in particular, it has yet to be elucidated whether polymer-membrane interactions and hole formation are responsible for endosomal escape and how such process can be controlled to optimize gene cytoplasmic delivery.

\section{Molecular simulation of DNA complexation in an endosome.}

To check whether protonation increased of a PEI chain is associated with endosomal swelling disruption, we summarize here some recent findings [58] about complexation between two oppositely charged polymers. Other theoretical studies [59, 60] have shown that after complexation, extended oppositely charged polymer chains collapse into a compact globule, even though the initial chain configuration contained bound ions. Ions are progressively released by polycation-polyanion pairs attraction and the final polymer complex, containing two chains separated from the rest of the ions.

In the presence of many interacting ions, the dynamics of a DNA molecule mixed with polycations is quite complex. But motivated by experimental data [61] suggesting that the number of polycations can affect the PEI-DNA interaction, we recently used Brownian simulations [58] to study the effect of various proton concentrations on this interaction. We modeled polymers as flexible bead-spring chains, where each bead represents a charged monomer, while ions are modeled as charged particles. The solvent is treated as a dielectric continuum with dielectric a constant $\epsilon=80$. We started with two oppositely charged chains whose monomer positions are respectively

$$
\begin{aligned}
& \boldsymbol{x}=\left(\boldsymbol{x}_{1}, \cdots, \boldsymbol{x}_{N}\right), \\
& \boldsymbol{y}=\left(\boldsymbol{y}_{1}, \cdots, \boldsymbol{y}_{M}\right),
\end{aligned}
$$

mixed with ions at position

$$
\boldsymbol{z}=\left(\boldsymbol{z}_{1}, \cdots, \boldsymbol{z}_{P}\right)
$$

where the charge electro neutrality is preserved $P=M+N$. The motion of a polymer chain in an overdamped medium is described by the Smoluchowski's limit of the Langevin equation, which reduces to a system of first order stochastic differential equations. The dynamics of bead $i$ at position $\boldsymbol{x}_{i}$ in a potential $U(\boldsymbol{x}, \boldsymbol{y}, \boldsymbol{z})$ is

$$
\dot{\boldsymbol{x}}_{i}+\frac{1}{\gamma} \frac{\partial U}{\partial \boldsymbol{x}_{i}}=\sqrt{2 D} \dot{\boldsymbol{w}}_{i} \text { for } i=1 . . N
$$


where $D$ is the diffusion coefficient and $\dot{\boldsymbol{w}}_{1}, . . \dot{\boldsymbol{w}}_{N}$ are N-independent 3-dimensional Brownian motions and $\gamma$ is the viscosity coefficient. The total potential $U=U(\boldsymbol{x}, \boldsymbol{y}, \boldsymbol{z})$ is the sum of various potential terms

$$
U(\boldsymbol{x}, \boldsymbol{y}, \boldsymbol{z})=U_{E L}(\boldsymbol{x})+U_{E L}(\boldsymbol{y})+U_{\text {bend }}(\boldsymbol{x})+U_{\text {bend }}(\boldsymbol{y})+U_{L J}(\boldsymbol{x}, \boldsymbol{y}, \boldsymbol{z})+U_{C}(\boldsymbol{x}, \boldsymbol{y}, \boldsymbol{z})
$$

where

- $U_{E L}(\boldsymbol{x})$ is the polymer elastic energy: the potential well $U_{k}$ generated by two adjacent springs on the $k$-bead is the sum of the two neighboring potentials:

$$
\begin{gathered}
U_{k k+1}\left(\boldsymbol{x}_{k}, \boldsymbol{x}_{k+1}\right)=k\left(\frac{1}{2}\left|\boldsymbol{x}_{k}-\boldsymbol{x}_{k+1}\right|^{2}-l_{0}\left|\boldsymbol{x}_{k}-\boldsymbol{x}_{k+1}\right|\right) \\
U_{k k-1}\left(\boldsymbol{x}_{k}, \boldsymbol{x}_{k-1}\right)=k\left(\frac{1}{2}\left|\boldsymbol{x}_{k}-\boldsymbol{x}_{k-1}\right|^{2}-l_{0}\left|\boldsymbol{x}_{k}-\boldsymbol{x}_{k-1}\right|\right),
\end{gathered}
$$

where $k$ is the linear elasticity constant and $l_{0}$ the equilibrium length. For $0<k<N$, the potential $U_{k}$ is

$$
\begin{aligned}
U_{k}\left(\boldsymbol{x}_{k-1}, \boldsymbol{x}_{k}, \boldsymbol{x}_{k+1}\right) & =U_{k k+1}\left(\boldsymbol{x}_{k}, \boldsymbol{x}_{k+1}\right)+U_{k k-1}\left(\boldsymbol{x}_{k}, \boldsymbol{x}_{k-1}\right) \\
U_{0}\left(\boldsymbol{x}_{1}, \boldsymbol{x}_{2}\right) & =U_{12}\left(\boldsymbol{x}_{1}, \boldsymbol{x}_{2}\right) \\
U_{N}\left(\boldsymbol{x}_{N-1}, \boldsymbol{x}_{N}\right) & =U_{N N-1}\left(\boldsymbol{x}_{N}, \boldsymbol{x}_{N-1}\right)
\end{aligned}
$$

and the total potential is given by

$$
U_{E L}(\boldsymbol{x})=\sum_{k=1}^{N} U_{k}\left(\boldsymbol{x}_{k-1}, \boldsymbol{x}_{k}, \boldsymbol{x}_{k+1}\right)
$$

The elastic energy $U_{E L}(\boldsymbol{y})$ of the second chain is computed similarly.

- $U_{L J}(\boldsymbol{x})$ is the repulsive Lennard-Jones (LJ) potential, which models the excluded volume between all the particles in the system (monomers and ions) and it is computed as follows: the LJ-potential between two monomers of the same chain is given by

$$
U_{L J}\left(\boldsymbol{x}_{i}, \boldsymbol{x}_{j}\right)= \begin{cases}4 \epsilon_{L J}\left[\left(\frac{\sigma}{\boldsymbol{x}_{i j}}\right)^{12}-2\left(\frac{\sigma}{\boldsymbol{x}_{i j}}\right)^{6}\right]+\epsilon_{L J} & \boldsymbol{x}_{i j} \leq \boldsymbol{x}_{c} \\ 0 & \boldsymbol{x}_{i j}>\boldsymbol{x}_{c}\end{cases}
$$

where $\boldsymbol{x}_{i j}=\left|\boldsymbol{x}_{i}-\boldsymbol{x}_{j}\right|$ is the distance between the monomers, $\epsilon$ is the depth of the potential well, $\sigma$ is the (finite) distance at which the inter-particle potential is zero. The LJ-potential is cut off at a distance $\boldsymbol{x}_{c}=2^{1 / 6} \sigma$ and the constant $\epsilon_{L J}$ is added to avoid discontinuity at $\boldsymbol{x}_{c}$.

A similar potential is used to compute the LJ-interaction between monomers of the second chain $U_{L J}\left(\boldsymbol{y}_{i}, \boldsymbol{y}_{j}\right)$, monomers belonging to different chains $U_{L J}\left(\boldsymbol{x}_{i}, \boldsymbol{y}_{j}\right)$, monomers 
and ions, $U_{L J}\left(\boldsymbol{x}_{i}, \boldsymbol{z}_{j}\right)$ and $U_{L J}\left(\boldsymbol{y}_{i}, \boldsymbol{z}_{j}\right)$, ion-ion $U_{L J}\left(\boldsymbol{z}_{i}, \boldsymbol{z}_{j}\right)$, so that the total LJ potential is given by:

$$
\begin{aligned}
U_{L J}(\boldsymbol{x}, \boldsymbol{y}, \boldsymbol{z})= & \sum_{i \neq j} U_{L J}\left(\boldsymbol{x}_{i}, \boldsymbol{x}_{j}\right)+\sum_{i, j} U_{L J}\left(\boldsymbol{x}_{i}, \boldsymbol{y}_{j}\right)+\sum_{i, j} U_{L J}\left(\boldsymbol{x}_{i}, \boldsymbol{z}_{j}\right)+ \\
& +\sum_{i \neq j} U_{L J}\left(\boldsymbol{y}_{i}, \boldsymbol{y}_{j}\right)+\sum_{i, j} U_{L J}\left(\boldsymbol{y}_{i}, \boldsymbol{z}_{j}\right)+\sum_{i \neq j} U_{L J}\left(\boldsymbol{z}_{i}, \boldsymbol{z}_{j}\right)
\end{aligned}
$$

- The stiffness of a polymer is described by a bending energy of the form

$$
U_{\text {bend }}(\boldsymbol{x})=\frac{\kappa_{\text {ang }}}{2} \sum_{i=2}^{N}\left(\boldsymbol{x}_{i-1}-2 \boldsymbol{x}_{i}+\boldsymbol{x}_{i+i}\right)^{2}=\kappa_{\text {ang }} \sum_{i=1}^{N-1}\left(1-\boldsymbol{u}_{i} \cdot \boldsymbol{u}_{i+1}\right),
$$

where $\boldsymbol{u}_{\boldsymbol{i}}=\left(\boldsymbol{x}_{i+1}-\boldsymbol{x}_{i}\right) /\left|\boldsymbol{x}_{i+1}-\boldsymbol{x}_{i}\right|$ is the unit bond vector connecting two consecutive monomers and $\boldsymbol{x}_{i}$ is the position of the $i-t h$ monomer. This potential depends on the angle $\theta_{i}$ between two successive monomers since $\boldsymbol{u}_{i} \cdot \boldsymbol{u}_{i+1}=\cos \theta_{i}$. The constant $\kappa_{\text {ang }}$ measures the bending rigidity which is related to the persistence length of the polymer by $l_{p}=\kappa_{a n g} /\left(k_{B} T\right)$, where $k_{B}$ is the Boltzmann constant and $T$ is the temperature 62]. The persistence length characterize the stiffness of a polymer and it is defined as follows:

$$
\langle\cos \theta\rangle \sim \exp \frac{l}{l_{P}}
$$

where $\theta$ is the angle between two vectors tangent to the polymer separated by a distance $l$, and the cosines of the angles is averaged (angled brackets) over all configurations. The stiffness energy $U_{\text {bend }}(\boldsymbol{y})$ of the second chain is similarly computed.

- The electrostatic potential between two charged monomers (charges are relocalized at the monomer) at position $\boldsymbol{x}_{i}$ and $\boldsymbol{x}_{j}$ is given by the Coulomb potential

$$
U_{C}\left(\boldsymbol{x}_{i}, \boldsymbol{x}_{j}\right)=\frac{e^{2} Z_{i} Z_{j}}{4 \pi \epsilon} \sum_{i \neq j} \frac{1}{\left|\boldsymbol{x}_{i}-\boldsymbol{x}_{j}\right|}
$$

where $\epsilon$ is the dielectric constant of the medium and $Z_{i}$ the valence of the ion $i$. The total electrostatic potential is

$$
\begin{aligned}
U_{C}(\boldsymbol{x}, \boldsymbol{y}, \boldsymbol{z})= & \sum_{i \neq j} U_{C}\left(\boldsymbol{x}_{i}, \boldsymbol{x}_{j}\right)+\sum_{i, j} U_{C}\left(\boldsymbol{x}_{i}, \boldsymbol{y}_{j}\right)+\sum_{i, j} U_{C}\left(\boldsymbol{x}_{i}, \boldsymbol{z}_{j}\right)+ \\
& +\sum_{i \neq j} U_{C}\left(\boldsymbol{y}_{i}, \boldsymbol{y}_{j}\right)+\sum_{i, j} U_{C}\left(\boldsymbol{y}_{i}, \boldsymbol{z}_{j}\right)+\sum_{i \neq j} U_{C}\left(\boldsymbol{z}_{i}, \boldsymbol{z}_{j}\right),
\end{aligned}
$$

which represents the total Coulomb interactions between monomers on a single chain, $U_{C}\left(\boldsymbol{x}_{i}, \boldsymbol{x}_{j}\right)$ and $U_{C}\left(\boldsymbol{y}_{i}, \boldsymbol{y}_{j}\right)$. For monomers located on different chains the potential is $U_{C}\left(\boldsymbol{x}_{i}, \boldsymbol{y}_{j}\right)$, for monomer-ion interaction, it is $U_{C}\left(\boldsymbol{y}_{i}, \boldsymbol{z}_{j}\right)$ and $U_{C}\left(\boldsymbol{y}_{i}, \boldsymbol{z}_{j}\right)$, and finally the ion-ion interaction is given by $U_{C}\left(\boldsymbol{z}_{i}, \boldsymbol{z}_{j}\right)$. 
The dynamics of the second polymer with $M$ beads and positions $\boldsymbol{y}=\left(\boldsymbol{y}_{1}, \cdots, \boldsymbol{y}_{M}\right)$ is governed by

$$
\dot{\boldsymbol{y}}_{i}+\frac{1}{\gamma} \frac{\partial U}{\partial \boldsymbol{y}_{i}}=\sqrt{2 D} \dot{\boldsymbol{w}}_{i} \text { for } i=1 . . M
$$

Finally the ions with positions $\boldsymbol{z}=\left(\boldsymbol{z}_{1}, \cdots, \boldsymbol{z}_{M}\right)$ satisfy

$$
\dot{\boldsymbol{z}}_{i}+\frac{1}{\gamma} \frac{\partial U}{\partial \boldsymbol{z}_{i}}=\sqrt{2 D} \dot{\boldsymbol{w}}_{i} \text { for } i=1 . . P
$$

The previous stochastic equations were solved numerically [58], by standard Euler's scheme. The results of the simulations are presented in the next section.

\section{Numerical simulations of DNA-polymer interactions}

To check whether increasing the proton concentration modulates the DNA-PEI interaction and leads to the deformation of the DNA-polycation complex, we ran various Brownian simulations [58], that we summarize now. We initially placed two oppositely charged chains of total length $L_{p}$ in a 3D-cube of length $L$ and each monomer position is randomly chosen at equilibrium (the two spherical angles between monomers are uniformly distributed in the 2 -sphere $S(2)$ of radius $l_{0}$ ). To ensure electroneutrality, we added a number of ions, equal to the difference between the DNA and polycation charges. Periodic boundary conditions are imposed on the cube. $L$ was chosen such that the density $\rho=\left(L_{p} / L\right)^{3} \approx 10^{-4}$ is small. In that regime, there are no self-interactions between polymers. In a first transient regime, we waited for each chain to relax to equilibrium. Then, we move the chains close enough and restart the simulations to evaluate their interaction. We found that the structure of the final complex made of the two chains, after equilibrium is that of a flexible polycation winding around the DNA chain (Fig,4) and ions are uniformly distributed over the simulation box. To further study the consequences of decreasing the $\mathrm{pH}$ and changing the PEI ionization (accounting for the experimental procedure [63]), we repeated the previous simulations in the presence of different ionic concentrations (Fig. 4).

We further run additional simulations in a reflecting sphere, modeling an endosomal compartment to estimate the distribution of charges along the PEI monomers. We used [58] a Metropolis Monte-Carlo simulation [64] to evaluate the protonation configuration of a polymer having $N$ monomers. Each monomer is capable of binding a proton. To estimate this distribution, we compute the free energy at equilibrium by finding the minimum of

$$
G(\mathbf{x})=\sum_{i}^{N}\left(x_{i} k_{B} T \log 10\left(p H-p K_{i}^{0}\right)\right)+\frac{1}{2} \sum_{i \neq j} W_{i j} q_{i} q_{j}
$$

where $\mathbf{x}=\left\{x_{1}, x_{2}, \cdots, x_{N}\right\}$ and $x_{i}=1$ when site $i$ is protonated, 0 otherwise. The first term in 17 is the energy required to protonate a site, depending on $\epsilon$, the $\mathrm{pH}$ and the $p K$ of the PEI [64] and $W_{i j}$ is the electrostatic Coulomb interaction energy between sites $i$ and $j$ 
when they are both charged. Thus, the average protonation of a given site $x_{i}$ is obtained by averaging (Boltzmann weighted sum) over all configurations:

$$
\left\langle x_{i}\right\rangle=\frac{\sum_{x_{i}} x_{i} \exp ^{-G(x) / k_{B} T}}{\sum_{x_{i}} \exp ^{-G(x) / k_{B} T}}
$$

We used [58] the Metropolis algorithm [65] to sample the most probable states of $x_{i}$ and Fig 3 summarizes the configuration distributions for various $\mathrm{pH}$. At physiological concen-

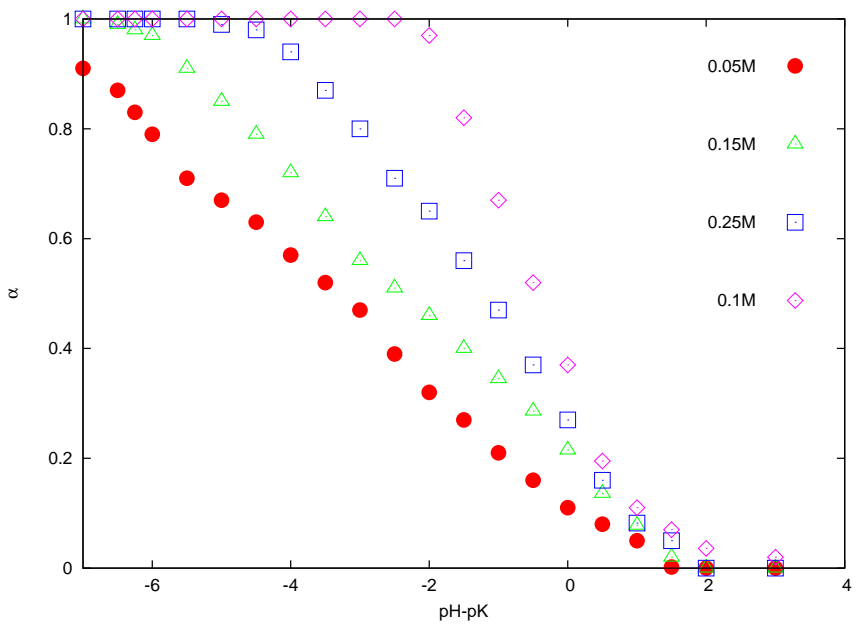

Figure 3: Simulated protonation curves of PEI for different salt concentrations, $0.01 \mathrm{M}, 0.05 \mathrm{M}, 1.0 \mathrm{M}$. At high salt concentration, there is a steep rise in the protonation as the $\mathrm{pH}$ approaches $p K_{0}$ and the chain is fully protonated for $p H-p K_{0} \sim 2$. As the salt concentration decreases, protonation of the chain becomes more difficult, because there are fewer ions to screen the electrostatic repulsion of charges on the chain.

tration $C_{s}=0.15 M$ and $p H=7.4$, then $p H-p K \simeq 2.7$, that is $50 \%$ of monomers are protonated, higher than experimental measurements (20\% of protonated sites [66]). DNAPEI complex seems to evolve to different configurations depending on the $\mathrm{pH}$. In Fig. 4 a, the DNA-polycation forms a complex. The polycation is only partially charged (indeed $20 \%$ of the amine groups are protonated [66]). The polycation behaves almost like a flexible chain, while DNA monomers are subject to strongest Coulomb repulsion making it stiffer and more extended, and almost immobile during complexation. Fig. 4b and 4c show the DNA/PEI complex configurations respectively at $\mathrm{pH}=6$ and 5 , during the endosomal acidification. Increased protonation could induce swelling [63] of the complex and consequently promoting endosome rupture. The result in [58] predicts that increasing attraction between oppositely charged polyelectrolytes lead to a more compact complex structure. Upon increasing the protonation state of the PEI, the positively charged chain condenses more tightly on the DNA, which become more flexible because its charged are now screened. The resulting complex resembles a typical toroid configuration found for DNA interacting with multivalent ions [67]. The same polyplex structure was previously obtained [68] by solving numerically modified Poisson-Boltzmann equations for the electrostatic potential and the polymer concentration. These results [58] suggest that two oppositely charged polymers condense upon lowering $\mathrm{pH}$ 


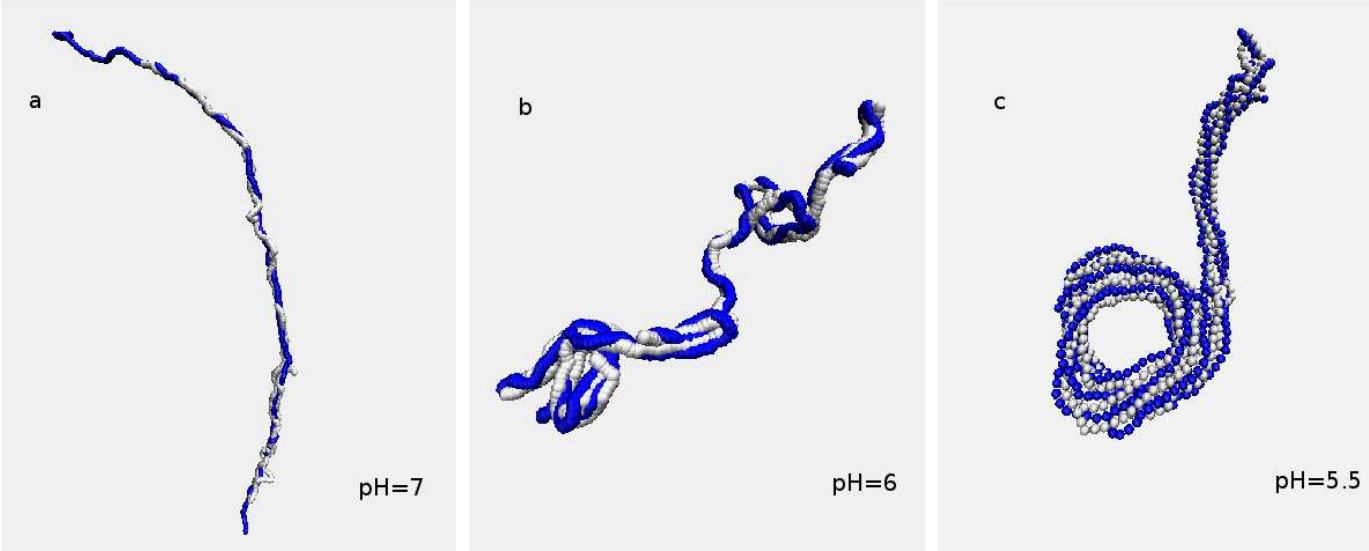

Figure 4: Snapshots of the equilibrium configurations of the DNA/PEI complex at $\mathrm{pH}$ $=7.0$ (left), $\mathrm{pH}=6.0$ (middle), $\mathrm{ph}=5.5$ (right). During the acidification process the size of the complex decreases. Each image represents the configuration at fixed $\mathrm{pH}$ after $10^{6}-10^{7}$ steps of the Brownian dynamics, which corresponds roughly to $1-10 s$.

and thus this mechanism cannot be responsible for the swelling of the endosome and endosomal membrane disruption. We shall now review another simulation sets, aimed to better

a

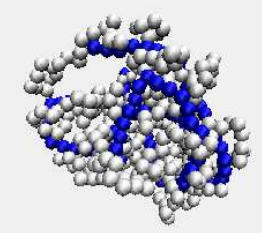

$\mathrm{pH}=7$ b

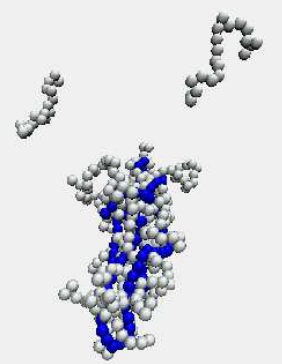

$\mathrm{pH}=6$

C

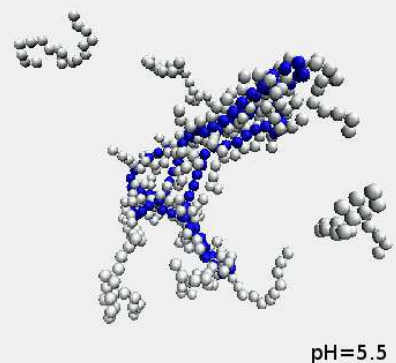

Figure 5: Snapshots of the equilibrium configurations for a DNA molecule complex with many small polycations, $\mathrm{pH}=7.0$ (left), $\mathrm{pH}=6.0$ (middle), $\mathrm{ph}=5.5$ (right). During the acidification process, the complex-DNA can swell due to an increased Coulomb repulsion and consequently some polycations can be released from the complex. Each frame represents the configuration at fixed $\mathrm{pH}$ after $10^{6}-10^{7}$ steps of the Brownian dynamics.

characterize an experimental procedure where the DNA molecule is mixed in solution with a fixed concentration of PEI, where the ratio $r=N / P$ of the $\mathrm{N}$ amine to $\mathrm{P}$ phosphate groups is fixed. A value of $N / P=4-10$ has been reported from experiments [61] and we simulate a DNA molecule with $N_{1}=100$ monomers when it is complexed with 20 polyactions, each of length $N_{2}=20$, making the ratio $r=N / P=4$. We use the same numerical method as described above with many polycations. Fig. 5 a shows the equilibrium configuration of the DNA complexed with the small polycations at physiological $\mathrm{pH}$ with periodic boundary 
conditions, while Fig $5 \mathrm{~b}$ and $5 \mathrm{c}$ represent the influence of acidification in a confined spherical microdomain. At physiological pH, the DNA complex is less compacted and more flexible due to an excess of positive charges resulting in additional screening, compared with the situation of two chains only (Fig $4 \mathrm{a}$ ). During acidification, at higher charge fraction of the PEI, some polycations are released from the complex and are free to diffuse in the endosome. The presence of free polycations has been also observed experimentally [69]. Recent experiments [70] have shown that purified polyplexes without free PEIs were less efficient in transfection compared to non-purified polyplexes. Hence interaction of free PEIs with the endosomal membrane, could play a role in endosomal escape, however a detailed model of membrane destabilizaton due to membrane-polymer interaction is currently missing.

In summary, Brownian simulations can be used to study processes occurring at intermediate scale between molecular and cellular. We have reviewed here some approaches method to simulate the interaction of oppositely charged complex polymers in endosomal following protonation. We reported that surprisingly, the protons and chloride endosomal influx which lead to an increase in the ionic concentrations and the enlargement of the polycation-DNA complex (due to internal charge repulsion), in the range of parameters such as $p H \in[5.5-7]$, the number of poycations from 1 to 20 [58], seems not to have any direct role in the endosome disruption. This result suggests that more refined scenario should be considered to study the direct PEI-DNA interaction during endosomal membrane disruption.

\section{Modeling the endosomal step of viral infection}

To better understand how viruses can be used as gene vectors, we now focus on one of the fundamental step of early infection, where the viral particles travel inside an endosome. Indeed, most viruses enter cells in an endosomal compartment, after binding to specific membrane receptors. To undergo cytoplasmic or nuclear replication [71, 72], and to avoid degradation in acidic lysosomes, viruses must then successfully escape the endosome. Enveloped viruses, such as Influenza, contain membrane-associated glycoproteins mediate the fusion between the viral and endosomal membranes. In particular, acidification of the endosome triggers the conformational change of the influenza hemagglutinins (HA) into a fusogenic state, leading to endosome-virus membranes fusion and genes release inside the cytoplasm.

Developing a biophysical model of the influenza endosomal step offers a general framework to study the influence of several parameters such as the endosomal size, the number of viral particles or the molecular structure of glycoproteins on the efficiency of viral escape. In particular, fusogenic peptides derived from viral glycoproteins are increasingly used in cationic synthetic vectors [8, 9], and the $\mathrm{pH}$-sensitivity of fusogenic glycoproteins can now be tuned by modifying the electrostatic stability of the fusogenic complex [73]. These engineered glycoproteins shall serve to design efficient gene vectors and quantitative models will help optimizing glycoprotein molecular properties with respect to the endosomal escape efficacy of the vector.

We shall review previous models [74] aimed to estimate the residence time of a viral particle inside an endosomal compartment. These models are based on considering the 
accumulation of discrete proton binding events, leading to the conformational change of HAs. This is the limiting step of genes release in the cytoplasm and the model was built into two steps: first, by fixing the concentration of protons, we used a Markov jump analysis to estimate the mean time for protons to bind the HA binding sites until a threshold is reached, triggering its conformational change into a fusogenic state. Interestingly, our analysis allow to extract from the HA conformational change kinetics measured experimentally at different $\mathrm{pH}[75$ the binding rates and the number of bound sites that are needed for the protein to change conformation. Second, coupling the $\mathrm{pH}$-dependent conformational change of HA glycoproteins with a linear proton influx rate, we analyzed the endosomal escape dynamics of influenza viruses. We predicted [74] that the size of the endosome drastically impacts both the escape kinetics and $\mathrm{pH}$, which reconciles different experimental observations: while a virus can escape from small endosomes (radius of $80 \mathrm{~nm}$ ) in the cell periphery at a $\mathrm{pH} \sim 6$ in about 10 minutes [76], it can also be routed towards the nuclear periphery, where escape from larger endosomes (radius of $400 \mathrm{~nm}$ ) is rapid (less than one minute) at pH 5 [77]. We shall now review in details the modeling of these two steps.

\section{Modeling the conformational change of glycoproteins}

To estimate the conformational change rate of a single HA glycoprotein at a given proton concentration $c$, we considered [74] that the protein changes conformation instantaneously when the number of bound sites reaches a critical threshold $n_{c r i t} \leq n_{s}$, where $n_{s}$ is the total number of HA binding sites.

To follow the time dependent number of protonated sites, we use a stochastic analysis based on Markov jump processes [78, 79, 80, 81], where during time $t$ and $t+\Delta t$, the amount of protonated sites $X(t, c)$ can either increase with a probability $r(X, c) \Delta t$ when a proton binds to a free site, decreases with probability $l(X, c) \Delta t$ when a proton unbinds or remains unchanged with probability $1-l(X, c) \Delta t-r(X, c) \Delta t$. Using a scaled variable $x(t, c)=\epsilon X(t, c)$ where $\epsilon=1 / n_{s}$ and $\Delta x=x(t+\Delta t, c)-x(t, c)$, the transition probabilities satisfy

$$
\begin{array}{r}
\operatorname{Pr}\{\Delta x=\epsilon \mid x(t, c)=x\}=r(x, c) \Delta t, \\
\operatorname{Pr}\{\Delta x=-\epsilon \mid x(t, c)=x\}=l(x, c) \Delta t, \\
\operatorname{Pr}\{\Delta x=0 \mid x(t, c)=x\}=(1-r(x, c)-l(x, c)) \Delta t .
\end{array}
$$

For a fixed proton concentration $c$, the transition probability function $p(y, t \mid x, c)$ that the proportion $x(t, c)$ of protonated sites is equal to $y$ at time $t, x(t, c)=y$, given that $x(0, c)=x$ is solution of the backward master equation [78, 79, 80, 81]:

$$
\begin{aligned}
p(y, t \mid x, c) & =p(y, t-\Delta t \mid x+\epsilon, c) r(x, c) \Delta t+p(y, t-\Delta t \mid x-\epsilon, c) l(x, c) \Delta t \\
& +p(y, t-\Delta t \mid x, c)(1-r(x, c) \Delta t-l(x, c) \Delta t),
\end{aligned}
$$


which can be expanded as (Kramers-Moyal expansion)

$$
\begin{aligned}
\frac{\partial p}{\partial t} & =L_{x} p=r(x, c) \sum_{n=1}^{\infty} \frac{\epsilon^{n}}{n !}\left(\partial_{x}\right)^{n} p(y, t \mid x, c) \\
& +l(x, c) \sum_{n=1}^{\infty} \frac{(-\epsilon)^{n}}{n !}\left(\partial_{x}\right)^{n} p(y, t \mid x, c) .
\end{aligned}
$$

The first time $\tau$ a glycoprotein is filled up to a critical threshold $x_{\text {crit }}=n_{\text {crit }} / n_{s}$ is the first passage time for the bound protons $x(t, c)$ to reach the level $x_{c r i t}$. The mean first passage time $\tau(x, c)$ is defined as the conditional expectation $\tau(x, c)=E[\tau \mid x(t=0, c)=x]$, and satisfies [81, 82]:

$$
\begin{aligned}
& L_{x} \tau(x, c)=-1 \text { for } x \text { in }\left[0, x_{\text {crit }}\right] \\
& \tau(x, c)=0 \text { for } x=x_{\text {crit }} \text { and } \frac{\partial \tau(x, c)}{\partial x}=0 \text { for } x=0 .
\end{aligned}
$$

We approximate the conformational change mean time with $\tau_{0}(c)=\tau\left(x_{0}(c), c\right)$, where $0<$ $x_{0}(c)<x_{c r i t}$ is the mean number of bound protons (concentration $c$ ), the leading order in $\epsilon \ll 1$ [78, 79, 80, 81] is

$$
\tau_{0}(c) \approx C(\epsilon, c)\left(1-\left(\frac{l\left(x_{c r i t}, c\right)}{r\left(x_{c r i t}, c\right)}\right)^{-\left(x_{c r i t}-x_{0}(c)\right) / \epsilon}\right)
$$

where

$$
C(\epsilon, c) \approx \frac{1}{r\left(x_{0}(c), c\right)} \frac{\sqrt{\frac{2 \pi}{\epsilon \frac{d}{d x}(l / r)\left(x_{0}(c), c\right)}}}{\phi\left(x_{c r i t}, c\right)}
$$

and

$$
\phi(x, c)=\frac{l(x, c) / r(x, c)-1}{\sqrt{l(x, c) / r(x, c)}} e^{-\frac{1}{\epsilon} \int_{x_{0}(c)}^{x} \log (l(s, c) / r(s, c)) d s} .
$$

Formula (21) relates the conformational change mean time of a single HA at a fixed protons concentration $c$ with the binding/unbinding rates $r(x, c)$ and $l(x, c)$ [74]

$$
r(x, c)=K c n_{s}(1-x), \text { and } l(x, c)=l(x)=K n_{s}(1-x) 10^{-(3(1-x)+4)},
$$

obtained from the mean number $x_{0}(c)$ of HA protonated sites at different pHs [83]. In addition, $n_{s}=9$ is the number of binding sites [83], $c$ the concentration of free protons in the endosome and $K$ the proton binding rate to free binding sites. Using these transition rates in formula (21), and comparing the theoretical conformational change mean time $\tau_{0}(c)$ with experimental data [75], we found that $K \approx 7.5 * 10^{3} \mathrm{~L}_{\mathrm{mol}} \mathrm{mol}^{-1} \mathrm{~s}^{-1}$ and $x_{\text {crit }} \approx 0.7$ [74]. Interestingly, the theoretical curve $\tau_{0}(c)$ describes the entire range of experimental data set of the influenza hemagglutinin [75], confirming the validity of our approach. Finally, 
comparing the mean proton binding time $\tau=1 /(K c)$ with the mean time $\tau_{d}$ for a proton to find a binding site by diffusion [84], we found [74] that:

$$
\tau_{d} / \tau \approx 10^{-4}
$$

suggesting that the HA binding time is dominated by a high activation barrier, which guarantees the stability of a conformational change, that cannot be easily triggered randomly (table 2 [75]). The theory predicts that the HA conformational changes occurs when roughly $x_{\text {crit }} n_{s} \approx 6 \mathrm{HA}$ binding sites are protonated.

\section{Modeling the endosomal escape of the influenza virus.}

During endosomal maturation, protons enter actively through V-ATPase pumps located in the endosomal membrane, leading to $\mathrm{pH}$ decrease and the conformational change of HAs into a fusogenic state [17]. After fusion of the viral and endosomal membrane, the influenza genes can be released inside the cytoplasm.

For a linear time dependent proton influx $\lambda t$, the the rate $\lambda$ is proportional to the number of pumps and thus to the endosomal surface [74]. Consequently, $\lambda$ may drastically increase as the endosome matures and increases its size by fusion of early endocytic vesicles into larger compartments [85]. As protons accumulate into the endosome, they can bind to influenza HAs, triggering their change of conformation when exactly 6 sites are protonated.

The entire viral membrane in contact with the endosome seems to fuse before genes escape, as observed in electron microscopy images (figure 5c [76]). Furthermore, another step of endosomal escape consists in the enlargement of the fusion pore, that should rely on the activation of additional HA located nearby the contact zone, between the virus and the endosome membranes [86. In previous models [74], we accounted for this complex cooperative mechanism between activated HAs nearby the contact zone, by considering that genes are released in the cytoplasm when the total number of activated HAs among the viral envelope reaches a threshold $0 \leq T \leq N_{H A}$, where $N_{H A}=400$ [87] is the total number of HAs covering a single virus. Consequently, the escape release time $\tau_{e}$ is defined by

$$
\tau_{e}=\inf \left\{t \mid H A_{6}(t) \geq T\right\} .
$$

where $H A_{0}(t), H A_{1}(t) \ldots H A_{6}(t)$ are the number of HAs that have bound $0,1 \ldots 6$ protons at time $t$. The acidification time course of an endosome containing an influenza virus is related to the number of free protons located in the endosome by $P(t)=\mathcal{N} V_{0} c(t)$, where $c(t)$ is the associated endosomal concentration at time $t$. Using the on-rate of a proton to a HA free binding site, $\tilde{r}(x)=r(x, P(t)) / P(t)=K n_{s}(1-x) /(\mathcal{N} V)$ and the off-rate $l(x)$ (22), the kinetics equations are

$$
\begin{array}{r}
H A_{0}+P \underset{l\left(1 / n_{s}\right)}{\stackrel{\tilde{r}\left(0 / n_{s}\right)}{\rightleftharpoons}} H A_{1} \\
H A_{1}+P \underset{l\left(2 / n_{s}\right)}{\stackrel{\tilde{r}\left(1 / n_{s}\right)}{\rightleftharpoons}} H A_{2} \\
H A_{5}+P \stackrel{\tilde{r}\left(5 / n_{s}\right)}{\longrightarrow} H A_{6}
\end{array}
$$


and the associated mass action law leads to the following differential equation system

$$
\begin{aligned}
\frac{d P(t)}{d t} & =\lambda-\sum_{i=0}^{5} \tilde{r}\left(\frac{i}{n_{s}}\right) P(t) H A_{i}(t)+\sum_{i=1}^{5} l\left(\frac{i}{n_{s}}\right) H A_{i}(t) \\
\frac{d H A_{0}(t)}{d t} & =-\tilde{r}\left(\frac{0}{n_{s}}\right) P(t) H A_{0}(t)+l\left(\frac{1}{n_{s}}\right) H A_{1}(t) \\
\frac{d H A_{1}(t)}{d t} & =\left(\tilde{r}\left(\frac{0}{n_{s}}\right) H A_{0}(t)-\tilde{r}\left(\frac{1}{n_{s}}\right) H A_{1}(t)\right) P(t)+l\left(\frac{2}{n_{s}}\right) H A_{2}(t)-l\left(\frac{1}{n_{s}}\right) H A_{1}(t) \\
\frac{d H A_{6}(t)}{d t} & =\tilde{r}\left(\frac{5}{n_{s}}\right) H A_{5}(t) P(t),
\end{aligned}
$$

where the proton influx rate in the endosome is a linear function of time $\lambda t$. The initial conditions $(t=0)$ are a neutral medium $p H=7$ and $P_{0}=\mathcal{N} V_{0} 10^{-7} \approx 1, H A_{0}(t=0)=$ $N_{H A}$ and $H A_{i}(t=0)=0$ for $1 \leq i \leq 6$.

The two critical unknown parameters of the model are the protons influx rate $\lambda$ and the mean number $T$ of activated HAs, needed for the large fusion pore formation allowing gene release inside the cytoplasm. These parameters were obtained by solving numerically the system of equations 26, and by comparing the time $\tau_{e}$ (formula 24) with the experimental mean escape time $(\approx 10$ minutes) obtained for the virus-endosome fusion in Hela cells [76]: we found 74

$$
T \approx 50 \% N_{H A}=200, \text { and } \lambda \approx 3 s^{-1} \text {. }
$$

\section{Using modeling approaches to study various endosomal pathways}

Viral fusion and genes release from small endosomes (radius $\sim 80 \mathrm{~nm}$ ) have been observed in cell periphery [76], starting only 10 minutes after viral entry. Although the endosomal $\mathrm{pH}$ in these small endocytic vesicles is not precisely known, it is believed to be $\approx 6$. However, most viruses are routed towards the nuclear periphery into larger endosomes (radius $\sim 450 \mathrm{~nm}$ ), where the escape time decreases to $1-2$ minutes and the associated escape pH to $\approx 5$ [77] (FIG. 6a).

To model the escape process in these two endosomal pathways, we developed a model [74] that accounts for the endosomal size, where the proton influx rate is scaled to the endosomal surface $\lambda(r)=\left(r / r_{0}\right)^{2} \lambda=(r /(80 n m))^{2} 3 s^{-1}$ [74]. Taking this scaling into account, we found by numerical resolution of equations (26) that the escape $\mathrm{pH}$ and mean escape time are drastically decreased as the endosome becomes larger. When the threshold to fusion is fixed to $T=200$ activated HAs [74], the escape $\mathrm{pH}$ is 5.8, and the escape time is 10 minutes for an endosomal radius of $80 \mathrm{~nm}$, while the escape time and the $\mathrm{pH}$ drop to $40 \mathrm{~s}$ and 5 respectively for a radius of $450 \mathrm{~nm}$ (Fig. 6b-c).

In the general context of cytoplasmic trafficking, we proposed [74] that, when viruses use the periphery pathway where they leave the endosome far away from the nucleus, at a $\mathrm{pH}$

6, they can further escape the rapid inactivation process occurring at $p H<5.4$ [89]. In that 
case, they still have to travel through the risky cytoplasm to a small nuclear pore to deliver their genetic material. However, for viruses using the other pathway leading directly to the perinuclear area, the escape endosomal $p H$ drops to $\leq 5$, thus this exposure leads to possible HA degradation resulting in greatly diminishing the escape capacity. Consequently, it is not clear which pathway is more efficient for viral gene delivery.
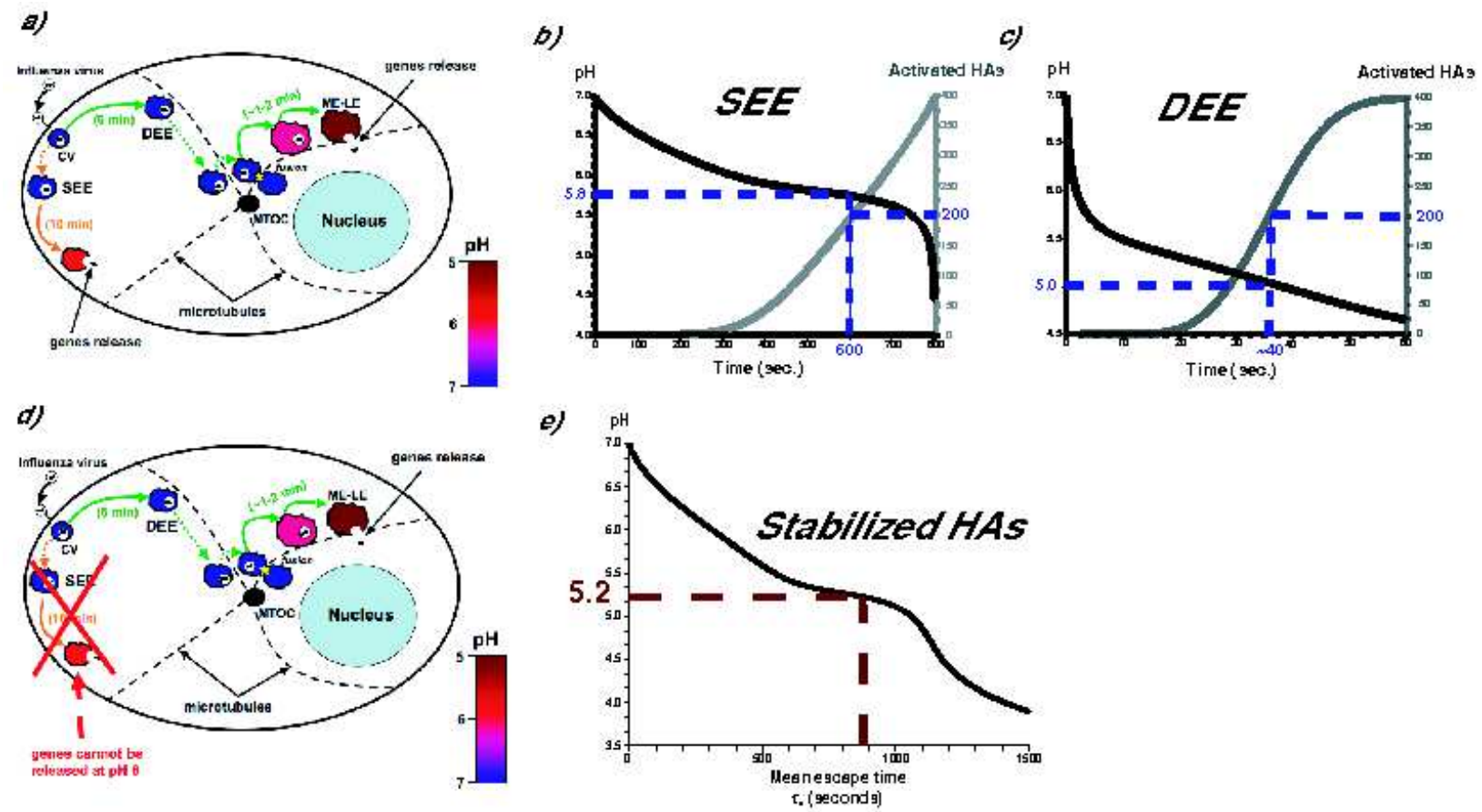

Figure 6: Schematic representation of the influenza virus endosomal pathways. Genes are released in the cytoplasm and the mean escape time and $\mathrm{pH}$ depends on the endosomal radius (a) After cell entry, the coated vesicle (CV) containing the virus can either be routed towards small static early endosome (SEE) in cell periphery [88, 76], where fusion occurs after 10 minutes at $\mathrm{pH} 6$ [76] (orange pathway), or it can fuse with dynamic early endosomes (DEE) traveling along microtubules, to reach the perinuclear region and rapidly fuse from larger endosomes (green pathway) [77, 88]. In the second case, fusion time is decreased to $1-2$ minutes and the escape $\mathrm{pH}$ drops to 5. (b) Time evolution of the number of activated HAs $H A_{6}(t)$ (grey line) and the endosomal pH (solid line) for a small SEE with $r=0.08 \mu m$ [76] (proton influx rate $\lambda=3 s^{-1}$ ). The $\mathrm{pH}$ is maintained above 5.8 for $H A_{6}(t) \leq 50 \% n_{H A}=200$ (highlighted with blue dotted lines) leading to genes release in the cytoplasm. (c) For a larger perinuclear DEE with $r=0.45 \mu m$ [85], the proton influx rate has been scaled to the endosome surface $\lambda=(45 / 8)^{2} 3 s^{-1}=95 s^{-1}$, which leads to a rapid gene release in about 40 seconds. d-e) Engineered Stabilized HAs with an increases low-pH stability shall prevent viral vectors escape from peripherical endosomes at $\mathrm{pH} 6$.

\section{Perspectives in developing simulations to optimize synthetic virus-like vectors}

For many enveloped viruses, conformational change of glycoproteins into a fusogenic conformation is triggered either by cumulative discrete binding of protons (e.g. class I, II and III fusogenic glycoproteins [90, 91]), or by low-pH activated proteases (e.g. Ebola GP protein 
and the SARS coronavirus spike protein S [92, 93]). In addition, fusogenic glycoproteins are increasingly used in many synthetic drug delivery systems such as siRNA delivery systems [94] or cationic synthetic gene vectors [8, 9]. Thus, the generic model developed in [74 for the influenza virus can be extended to this large class of viruses and synthetic vectors. Furthermore, the pH stability of influenza glycoproteins has been recently tuned by incorporating or removing electrostatic bonds into the fusogenic complex [73]. Consequently, developing biophysical models to quantify the endosomal escape kinetics and $\mathrm{pH}$ of viruses with respect to glycoproteins molecular properties is timely and could be further applied to optimize gene vector design. Indeed approaches developed in [74] can predict the endosomal time course of vectors or mutant viruses covered by these HA variants. In [74], we have predicted that using stabilized forms of HA glycoproteins [73] shifts the escape $\mathrm{pH}$ in small endosomes from 6 to 5, which may prevent vectors to use the peripherical endosomal pathway (figure 6 d-e). Consequently, if genes degradation inside the cytoplasm is a limiting barrier to transfection efficiency, we predict that stabilized mutant viruses may increase the ability of vectors to deliver genes to nuclear pores.

\section{Modeling gene carrier cytoplasmic dynamics}

We end this review by a section on quantifying the success of cytoplasmic trafficking. Indeed, following the endosomal escape, viral and synthetic gene vectors have to travel through the crowded and risky cytoplasm to reach the nucleus and deliver their genome through the nuclear pores. Recent imaging techniques now allow single particle tracking [95, 71, 96], which can be used to model cytoplasmic motion of gene vectors. Because gene vectors do not possess means of locomotion, they entirely rely on diffusion and active transport along MTs to reach the nucleus.

The cell cytoplasm is a highly crowded environment and diffusion of macromolecules depends on their size: while non interacting spherical particles with radius up to $\approx 25 \mathrm{~nm}$ are freely diffusible in the cell cytoplasm [97], increasing the size above $45 \mathrm{~nm}$ reduces considerably the motion [11]. Interestingly large viral particles have developed nuclear targeting signals to be actively transported along microtubules, which resulted in drastically decrease their arrival time to the nucleus [12]. It remains a challenging question to analyze their associated viral trajectories, which consist of a succession of free or confined diffusion and/or ballistic periods [95, 171, 96]. The analysis of such random trajectories starts with the position $\mathbf{X}(t)$ at time $t$ of the gene vector, which is a stochastic process [81, 98] and the dynamics depends on forces applied on the particle. When the motion of the gene vector is purely diffusive such as for cationic synthetic vectors, the overdamped equation for the velocity is simply $d \mathbf{X} / d t=\sqrt{2 D} d \mathbf{W} / d t$, where $\mathbf{W}$ is the standard Brownian motion. For a viral gene vector, the motion is usually assisted by active transport along MTs and the stochastic equation for the velocity becomes

$$
\frac{d \mathbf{X}}{d t}=\mathbf{b}(\mathbf{X})+\sqrt{2 D} \frac{d \mathbf{W}}{d t}
$$

where $\mathbf{b}$ is a drift that accounts for ballistic periods along MTs. This continuous Langevin description can be used to generate computer simulations of trajectories [81, 99] in free and 
confined environment [100, and is the basis to derive asymptotic formulas for the probability and the mean first passage time of the vector to a nuclear pore [84]. However, to reduce the complexity of large simulations, a first step consists in studying the dependency of $\mathbf{b}(\mathbf{X})$ as a function of the MTs organization and the viral dynamical properties (diffusion constant $D$, affinity with microtubules and net velocity along MTs) [101, 102].

\section{From the stochastic description of vector trajectories to the probability and the mean arrival time to a small nuclear pore.}

The crowded cytoplasm is a risky environment for gene vectors that can be either trapped or degraded through the cellular defense machinery. Consequently, the cytoplasmic trafficking is rate limiting for genes expression, and to analyze quantitatively that step, we derived [103, 104] asymptotic expressions for the probability $P_{n}$ and the mean time $\tau_{n}$ a single gene vector arrives to one of the $n$ small nuclear pores. In particular, obtaining these expressions allows to explore the phase space of parameters and in particular links global quantitative outputs measuring the success of nuclear genes delivery with the cellular geometry, the MTs organization and the dynamical properties of gene vectors. To obtain these expression, we modeled the viral degradation or immobilization by a steady state degradation rate $k(\mathbf{x})$. We then introduce the survival probability density function (SPDF) $p(\boldsymbol{x}, t)$, which is the probability to find a live (not degraded) viral particle inside a cytoplasmic volume element $\boldsymbol{x}+d \boldsymbol{x}$ at time $t[103$

$$
p(\boldsymbol{x}, t) d \boldsymbol{x}=\operatorname{Pr}\left\{X(t) \in \boldsymbol{x}+d \boldsymbol{x}, \tau^{k}>t, \tau^{a}>t \mid p_{i}\right\},
$$

where $\tau^{a}$ is the first time for a live virus to arrive to an absorbing nuclear pore, denoted $\partial N_{a}$ and $\tau^{k}$ the first time that it is degraded. The viral initial distribution is $p_{i}$. The SPDF $p(x, t)$ satisfies the Fokker-Planck equation (FPE) [81]

$$
\begin{aligned}
\frac{\partial p(\boldsymbol{x}, t)}{\partial t} & =D \Delta p(\boldsymbol{x}, t)-\nabla \cdot \boldsymbol{b}(\boldsymbol{x}) p(\boldsymbol{x}, t)-k(\boldsymbol{x}) p(\boldsymbol{x}, t) \quad \text { for } \quad \boldsymbol{x} \in \Omega \\
p(\boldsymbol{x}, 0) & =p_{i}(\boldsymbol{x}) \quad \text { for } \quad \boldsymbol{x} \in \Omega
\end{aligned}
$$

which describes the time evolution of the vector probability density function. The boundary conditions are

$$
\begin{array}{rl}
p(\boldsymbol{x}, t) & =0 \quad \text { for } \quad \boldsymbol{x} \in \partial N_{a} \\
\boldsymbol{J}(\boldsymbol{x}, t) \cdot \boldsymbol{n}_{\boldsymbol{x}}=0 & \boldsymbol{x} \in \partial \Omega-\partial N_{a}
\end{array}
$$

where the first condition describes the particle absorption at the nuclear pores area $\partial N_{a}$ and the second one the reflection of the vector on the remaining boundary area of the cell $\partial \Omega-\partial N_{a}$. The flux density vector $\boldsymbol{J}(\boldsymbol{x}, t)$ is defined by

$$
\boldsymbol{J}(\boldsymbol{x}, t)=-D \nabla p(\boldsymbol{x}, t)+\boldsymbol{b}(\boldsymbol{x}) p(\boldsymbol{x}, t),
$$

where $\boldsymbol{n}_{\boldsymbol{x}}$ is the unit outer normal at a boundary point $\boldsymbol{x}$. The probability $P_{n}$ that a live virus arrives to a nuclear pore and the conditional mean time can be expressed using the SPDF as

$$
P_{n}=\operatorname{Pr}\left\{\tau^{a}<\tau^{k}\right\}=\oint_{\partial N_{a}} \int_{0}^{\infty} \boldsymbol{J}(\boldsymbol{x}, t) \cdot \boldsymbol{n}_{\boldsymbol{x}} d S_{\boldsymbol{x}} d t
$$


and

$$
\begin{aligned}
\tau_{n}=E\left[\tau^{a} \mid \tau^{a}<\tau^{k}\right] & =\int_{0}^{\infty}\left(1-\operatorname{Pr}\left\{\tau^{a}<t \mid \tau^{a}<\tau^{k}\right) d t\right. \\
& =P_{n} \int_{0}^{\infty} \oint_{\partial N_{a}} t \boldsymbol{J}(\boldsymbol{x}, t) \cdot \boldsymbol{n}_{\boldsymbol{x}} d S_{\boldsymbol{x}} d t
\end{aligned}
$$

where $\operatorname{Pr}\left\{\tau^{a}<t \mid \tau^{a}<\tau^{k}\right\}$ is the conditional cumulative density function of the absorption time, given that the gene vector arrives alive to a nuclear pore. For $n$ identical nuclear pores modeled as absorbing disks of radius $\epsilon$ and when the drift is a gradient potential $(\mathbf{b}(\mathbf{x})=-\nabla \Phi(\boldsymbol{x}))$, the leading order term of $P_{n}$ and $\tau_{n}$ in $\epsilon$ have been estimated [103].

$$
P_{n}=\frac{e^{-\Phi_{0} / D}}{\frac{1}{4 D n \epsilon} \int_{\Omega} e^{-\Phi(\boldsymbol{x}) / D_{k}(\boldsymbol{x}) d \boldsymbol{x}+e^{-\Phi_{0} / D}}},
$$

and,

$$
\tau_{n}=\frac{\frac{1}{4 D n \epsilon} \int_{\Omega} e^{-\Phi(\boldsymbol{x}) / D} d \boldsymbol{x}}{\frac{1}{4 D n \epsilon} \int_{\Omega} e^{-\Phi(\boldsymbol{x}) / D} k(\boldsymbol{x}) d \boldsymbol{x}+e^{-\Phi_{0} / D}},
$$

where $\Phi_{0}$ is the constant value of the radial potential $\Phi(\boldsymbol{x})$ on the centered nucleus where the nuclear pores are uniformly distributed. These asymptotic results rely on the narrow escape theory [84], which is a general asymptotic method to estimate the mean first passage time of a Brownian motion confined in a domain to escape through a small opening.

As the number of nuclear pores $n$ remains small, the asymptotic validity of the previous expressions 36 agree in some limits with Brownian simulations [104]. However, these formulas do not account for the possible interactions between the small absorbing pores and indeed, $\lim _{n \rightarrow \infty, n \epsilon^{2} \ll 1}\langle\tau\rangle=0$. Because the nucleus contains a large number of nuclear pores $(n=2000$ [105]) that cover a small total area (around $1 \%$ of the nuclear surface), a refined analysis has been developed to account for the nuclear geometry [110]. In addition, interaction between absorbing windows can drastically affect the MFPT [106, 107].

Using electrostatic arguments, when the number of nuclear pores $n \gg 1$ is large and cover uniformly a small surface of the nucleus $\Sigma$, the leading order of the narrow escape time for a pure Brownian particle is 108

$$
\tau_{n}=\frac{|\Omega|}{D}\left(\frac{1}{C_{\Sigma}}+\frac{1}{4 n \epsilon}\right)
$$

where $C_{\Sigma}$ is the capacity of the nucleus (for a sphere of radius $\delta, C_{\Sigma}=4 \pi \delta$ ). When we neglected the geometrical interactions between the holes $1 / C_{\Sigma}=0$, the mean time (37) reduces to expression (36) for a Brownian particle $\phi=0$ with no killing activity $k=0$. Interestingly, both a quantitative and qualitative information can be derived from formula (37): the MFPT is shorter for a sphere covered with many small holes compared to a single 
large hole 104 with same surface. Actually, for a pure Brownian process, many absorbing small holes is equivalent to an almost totally absorbing surface [109].

Finally, in [110], we accounted for the interactions between absorbing nuclear pores, and obtained refined estimates for the probability $P_{n}$ and $\tau_{n}$ (formula 36), when the velocity is described by the stochastic equation (30)

$$
P_{n}=\frac{\frac{1}{|\partial \Sigma|} \oint_{\partial \Sigma} e^{-\Phi(\boldsymbol{x}) / D} d \boldsymbol{x}}{\frac{1}{|\partial \Sigma|} \oint_{\partial \Sigma} e^{-\Phi(\boldsymbol{x}) / D} d \boldsymbol{x}+\left(\frac{1}{4 n D \epsilon}+\frac{1}{D C_{\Sigma}}\right) \int_{\Omega} k(\boldsymbol{x}) e^{-\Phi(\boldsymbol{x}) / D} d \boldsymbol{x}}
$$

and

$$
\tau_{n}=\frac{\left(\frac{1}{4 n D \epsilon}+\frac{1}{D C_{\Sigma}}\right) \int_{\Omega} e^{-\Phi(\boldsymbol{x}) / D d \boldsymbol{x}}}{\frac{1}{|\partial \Sigma|} \oint_{\partial \Sigma} e^{-\Phi(\boldsymbol{x}) / D} d \boldsymbol{x}+\left(\frac{1}{4 n D \epsilon}+\frac{1}{D C_{\Sigma}}\right) \int_{\Omega} k(\boldsymbol{x}) e^{-\Phi(\boldsymbol{x}) / D} d \boldsymbol{x}} .
$$

Interestingly, for a biological cell with a spherical nucleus (radius $\delta=5 \mu m$ [105]), the $n=$ 2000 circular nuclear pores [105] (radius $\epsilon=25 n m$ [105]) cover a surface $\left(n \pi \epsilon^{2}\right) /\left(4 \pi \delta^{2}\right) \approx 1 \%$ of the total nuclear surface and $1 /(4 n D \epsilon)$ is only one third of $1 / C_{\Sigma}$. Using the parameter for the Adeno-Associated-Virus, with an affective diffusion constant $D=1.3 \mu m^{2} s^{-1}$ [95] and no degradation activity $(k=0)$, moving in a ball of radius $R=15 \mu \mathrm{m}$ ([11] ) containing a nucleus and a radial potential $\Phi(r)=0.2 r \mu^{2} m s^{-1}$ [104], using formula 39, we found that the time to a nuclear pore is $\tau_{n} \approx 1$ min., which is three time longer than the estimation using formula (36), when the effect of the nucleus is not taken into account.

\section{Conclusion}

Delivering a plasmid DNA in a cell is still a challenging task and a daunting hurdle of modern drug delivery methods. Being able to quantify cytoplasmic trafficking cannot only be used to optimize gene delivery, but also to design specific drug strategy against viral infection.

In the first part of the review, we summarized some recent progresses about quantifying the endosomal escape of synthetic polycations vectors, which defines a limiting step in gene delivery. It remains an open question to understand the precise mechanisms by which a plasmid is escaping and how the endosomal membrane is disrupted. We believe that any understanding can lead to a rational method to optimize the construction of polycations. In the second part, we presented some general modeling approaches to quantify cytoplasmic trafficking of enveloped viruses, such as influenza. It remains to find strategies to design these new synthetic gene vectors and for that goal, viruses appear as optimal models. Hybrid vectors made of a mixture of viral glycoproteins and polycations seem to be a promising direction and physical modeling and numerical simulations are certainly seducing tools to optimize the construction of such nano-scale carriers. 


\section{Acknowledgment:}

D. H. research is supported by an ERC-Starting Grant.

\section{References}

[1] Wikipedia http://en.wikipedia.org/wiki/Gene_therapy, and http://en.wikipedia.org/wiki/Gene_delivery

[2] S.J. Flint, L. W. Enquist, R. M. Krug, V. R. Racaniello, and A. M. Skalka, Principles of Virology: Molecular Biology, Pathogenesis, and Control, American Society Microbiology, 2003.

[3] M. Kokaia and A. Sorensen, Epilepsy and Gene therapy:Resculpturing Synpatic Transmissionwith Neuropeptides, in Encyclopedia of Basic Epilepsy Research ed. P A. Schwartzkroin, 2009.

[4] I.M. Verma, A tumultuous year for gene therapy, Mol. Ther., 2 (2000), pp. 415-416.

[5] P.L. Felgner, T.R. Gadek, M. Holm, R. Roman, H.W. Chan, M. Wenz, J.P. Northrop, G.M. Ringold, and M. Danielsen, Lipofection: a highly efficient, lipid-mediated DNA transfection procedure, Proc. Natl. Acad. Sci. USA, 84 (1987), pp.7413-7417.

[6] G.Y. Wu and C.H. Wu, Receptor-mediated in vitro gene transformation by a soluble DNA carrier system, J. Biol. Chem.,262 (1987), pp.4429-4432.

[7] O. Boussif, F. Lezoualch, M.A. Zanta, M.D. Megny, D. Scherman, B. Demeneix, and J.P. Behr, A versatile vector for gene and oligonucleotide transfer into cells in culture and in vivo: Polyethylenimine, Proc. Natl. Acad. Sci. USA, 92 (1995), pp.7297-7301.

[8] A. Abe, A. Miyanohara, and T. Friedmann, Enhanced gene transfer with fusogenic liposomes containing vesicular stomatitis virus G glycoprotein, J. Virol., 72 (1998), pp.61596163.

[9] Y. Tu, and J.S. Kim,A fusogenic segment of glycoprotein $H$ from herpes simplex virus enhances transfection efficiency of cationic liposomes, J. Gene Med.,10 (2008), pp.646654 .

[10] A.S. Verkman, Solute and macromolecule diffusion in cellular aqueous compartments, Trends Biochem. Sci., 27 (2002), pp.27-33.

[11] Dauty E., and A.S. Verkman, Actin cytoskeleton as the principal determinant of sizedependent DNA mobility in cytoplasm: a new barrier for non-viral gene delivery J. Biol. Chem., 280 (2005), pp.7823-7828.

[12] B. Sodeik, Mechanisms of viral transport in the cytoplasm, Trends Microbiol., 8 (2000), pp.465-472. 
[13] E. Neumann, M. Schaefer-Ridder, Y. Wang, and P.H. Hofschneider, Gene transfer into mouse lyoma cells by electroporation in high electric fields, EMBO J., 1 (1982), pp. 841-845.

[14] J. C. Weaver, and Y. A. Chizmadzhev, Theory of electroporation: A review, Biochemistry and Bioenergetics, 41 (1996), pp.135-160.

[15] K. C. Melikov, V. A. Frolov, A. Shcherbakov, A. V. Samsonov, Y. A. Chizmadzhev, and L. V. Chernomordik, Voltage-Induced Nonconductive Pre-Pores and Metastable Single Pores in Unmodified Planar Lipid Bilayer Biophys. J., 80 (2001), pp.1829-1836.

[16] G.A. Farr, L.G. Zhang, and P. Tattersall ,Parvoviral virions deploy a capsid-tethered lipolytic enzyme to breach the endosomal membrane during cell entry, Proc. Natl. Acad. Sci. U S A, 102 (2005), pp.17148-17153.

[17] J.J. Skehel and D.C. Wiley Receptor binding and membrane fusion in virus entry: the influenza hemagglutinin, Annu. Rev. Biochem., 69 (2000), pp.531-569.

[18] H. H. Chen, Y.P. Ho, X. Jiang, H. Mao, T. Wang and K.W. Leong Quantitative Comparison of Intracellular Unpacking Kinetics of Polyplexes by a Model Constructed From Quantum Dot-FRET Mol. Ther., 16 (2008), pp.324-332.

[19] J. Hansen, K. Qing, A. Srivastava, Infection of purified nuclei by adeno-associated virus 2, Mol. Ther., 4 (2001) pp. 289-296.

[20] P. L. Paine, L. C. Moore, and S. B. Horowitz, Nuclear envelope permeability, Nature, 254 (1975) pp.109-114.

[21] R. Peters, Fluorescence microphotolysis to measure nucleocytoplasmic transport and intracellular mobility, Biochim. Biophys. Acta, 864 (1986) pp.305-359.

[22] N. Pante, M. Kann, Nuclear pore complex is able to transport macromolecules with diameters of about $39 \mathrm{~nm}$, Mol. Biol. Cell, 13 (2002) pp.425-434.

[23] N.D. van Loo et al., Baculovirus infection of nondividing mammalian cells: mechanisms of entry and nuclear transport of capsids, J. Virol., 75 (2001) pp.961-970.

[24] Y. Suzuki, R. Craigie, The road to chromatin - nuclear entry of retroviruses, Nat. Rev. Microbiol., 5 (2007) 187-196.

[25] P. Palese, M.L. Shaw, Orthomyxoviridae: the viruses and their replication, in: P.M. Howley (Ed.), Fields Virology, fifth ed., Lippincott Williams \& Wilkins, Philadelphia (2007) pp. 1647-1740.

[26] L.C. Trotman et al. Import of adenovirus DNA involves the nuclear pore complex receptor CAN/Nup214 and histone H1, Nat. Cell Biol., 3 (2001) 1092-1100.

[27] E. Collins, J.C. Birchall, J.L. Williams, M. Gumbleton, Nuclear localisation and pDNA condensation in non-viral gene delivery, J. Gene Med., 9 (2007) pp.265-274. 
[28] A. R. Lowe, J. J. Siegel, P. Kalab, M. Siu, K. Weis, and J.T. Liphardt, Selectivity mechanism of the nuclear pore complex characterized by single cargo tracking, Nature, 467 (2010), pp.600-603.

[29] M. Stewart, Molecular mechanism of the nuclear protein import cycle, Nature Rev. Mol. Cell Biol. 8 (2007), pp.195-208.

[30] R. Peters, Functionalization of a nanopore: the nuclear pore complex paradigm, Biochim. Biophys. Acta, 1793 (2009) pp.1533-1539.

[31] S.R. Wente, M.P. Rout, The nuclear pore complex and nuclear transport, Cold Spring Harb. Perspect. Biol., 2 (2010) a000562.

[32] W.M. Gelbart, C.M. Knobler, Virology. Pressurized viruses, Science 323 (2009) pp.16821683

[33] G. Zuber, E. Dauty, M. Nothisen, P. Belguise, and J.P. Behr, Towards synthetic viruses, Adv. Drug Deliv. Rev., 52 (2001), pp.245-253.

[34] C.M. Varga, K. Hong, and D.A. Lauffenburger, Quantitative analysis of synthetic gene delivery vector design properties Mol. Ther., 5 (2001), pp.438-446.

[35] S. Yang, and S. May, Release of cationic polymer-DNA complexes from the endosome: A theoretical investigation of the proton sponge hypothesis, J. Chem. Phys., 129 (2008), pp.185105-185114.

[36] N.D. Sonawane, F.C. Szoka, and A.S. Verkman, Chloride accumualation and swelling in endosome enhances DNA transfer by polyamine-DNA polyplexes, J. Biol. Chem., 278 (2003), pp. 44826-44831.

[37] T. Bieber, W. Meissner, S. Kostin, A. Niemann, and H.P. Elsasser Intracellular route and transcriptional competence of polyethylenimine-DNA complexes, J. Cont. Rel., 82 (2002), pp.441-454.

[38] I.M Helander, K. Latva-Kala, and K. Lounatmaa, Permeabilizing action of polyethyleneimine on Salmonella typhimurium involves disruption of the outer membrane and interactions with I i popolysaccharide, Microbiol., 144 (1998), pp.385-390.

[39] I.M. Helander, H.L. Alakomi, K. Latva-Kala, and P. Koski, Polyethyleneimine is an effective permeabilizer of Gram-negative bacteria, Microbiol., 143 (1997), pp.3193-3199.

[40] N. Oku, S. Shibamoto, F. Ito, H. Gondo, and M. Nango, Low pH induced membrane fusion of lipid vesicles containing proton-sensitive polymer, Biochem., 26 (1987), pp.81458150 .

[41] A.R. Klemm, D. Young, and J.B. Lloyd, Effects of polyethyleneimine on endocytosis and lysosome stability, Biochem. Pharmacol., 56 (1998), pp.41-46. 
[42] P.R. Leroueil, S. A. Berry, K. Duthie, G. Han, V. M. Rotello, D.Q. McNerny, J. R. Baker, Jr., Bradford G. Orr, and M. B. Holl, Wide Varieties of Cationic Nanoparticles Induce Defects in Supported Lipid Bilayers, Nano Lett., 8 (2008), pp 420-424.

[43] M. Toner, and E. G. Cravalho, Kinetics and likelihood of membrane rupture during electroporation, Phys. Lett. A, 143 (1975),pp.409-412.

[44] H. Bermudez, H. Aranda-Espinoza, D. A. Hammer, and D. E. Discher, Pore stability and dynamics in polymer membranes, Europhys. Lett., 64 (2003),pp.550-556.

[45] O. Sandre, L. Moreaux, and F. Brochard-Wyart, Dynamics of transient pores in stretched vesicles, Proc. Natl. Acad. Sci. U.S.A., 96 (1999), pp.10591-10596.

[46] D. V. Zhelev, and D. Needham, Tension-stabilized pores in giant vesicles: determination of pore size and pore line tension, Biochim. Biophys. Acta, 1147 (1993), pp.89-104.

[47] J. D. Moroz, and P. Nelson, Dynamically-Stabilized Pores in Bilayer Membranes, Biophys. J., 72 (1997), pp.2211-2216.

[48] E. Karatekin, O. Sandre, and F. Brochard-Wyart, Transient pores in vesicles, Polym. Int., 52 (2003), pp.486-493.

[49] O. Farago, "Water-free" computer model for fluid bilayer membranes, J. Chem. Phys., 119 (2003), pp.596-605 .

[50] R. R. Netz, and M. Schick, Pore formation and rupture in fluid bilayers, Phys. Rev. E, 53 (1996), pp. 3875-3885.

[51] F. Brochard-Wyart, P. G. de Gennes, and O. Sandre, Transient pores in stretched vesicles: role of leak-out, Physica A, 278 (2000), pp.32-51.

[52] V. Talanquer, and D. W. Oxtoby, Nucleation of Pores in Amphiphile Bilayers, J. Chem. Phys.,118 (2003), pp.872-877.

[53] L. Fournier and B. Joos, Lattice model for the kinetics of rupture of fluid bilayer membranes, Phys. Rev. E, 67 (2003), 051908.

[54] Y. Levin and M. A. Idiart, Pore dynamics of osmotically stressed vesicles, Physica A, 331 (2004),pp. 571-578.

[55] O. Farago, Membrane fluctuations near a plane rigid surface, Phys. Rev. E, 78 (2008), pp.051919.

[56] P. Sens, and S. A. Safran, Pore formation and area exchange in tense membranes, Europhys. Lett., 43 (1998), pp.95.

[57] C. D. Santangelo, and O. Farago, Pore formation in fluctuating membranes, J. Chem. Phys., 122 (2005), pp.044901. 
[58] C.Amoruso, D. Holcman, Modeling the interaction of oppositely charged polyelectrolytes in an endosome (submitted).

[59] R. G. Winkler, M. O. Steinhauser, and P. Reineker, Complex formation in systems of oppositely charged polyelectrolytes: A molecular dynamics simulation study, Phys. Rev. E, 66 (2002), pp.021802.

[60] D. Srivastava, and M. Muthukumar, Interpenetration of interacting polyelectrolytes, Macromol., 27 (1994), pp.1461-1465.

[61] S. Choosakoonkriang, B. A. Lobo, G. S. Koe, J. G. Koe, and C. R. Middaugh, Biophysical characterization of PEI/DNA complexes, J. Pharm. Sci., 92 (2003), pp.1710-1722.

[62] L. D. Landau and E. M. Lifshitz, Theory of Elasticity Pergamon, (1986) New York.

[63] J.P. Behr, The proton sponge: a trick to enter cells the viruses did not exploit, Int. J. Chem., 51 (1997), pp. 34-36.

[64] P. Beroza, D.R. Fredkin, M.Y. Okamura, and G. Feher, Protonation of interacting residues in a protein by a Monte Carlo method: application to lysozyme and the photosynthetic reaction center of Rhodobacter sphaeroides, Proc. Natl. Acad. Sci. U.S.A, 88 (1991), pp.5804-5808.

[65] N. Metropolis, A.W. Rosenbluth, M.N. Rosenbluth, A.H. Teller, E. Teller, Equations of State Calculations by Fast Computing Machines. J. of Chem. Phys., 21 (1953) (6): pp. 10871092.

[66] Suh, J.; Paik, H. J.; Hwang, B. K. Bioorg. Chem. (1994), 22, pp. 318327.

[67] M. Stevens, Simple simulations of DNA condensation, Biophys. J., 80 (2001), pp. 130139.

[68] S. Yang, and S. May, Release of cationic polymer-DNA complexes from the endosome: A theoretical investigation of the proton sponge hypothesis, J. Chem. Phys., 29 (2008), pp. 185105.

[69] J.P. Clamme, J. Azoulay, and Y. Mely, Monitoring of the Formation and Dissociation of Polyethylenimine/DNA Complexes by Two Photon Fluorescence Correlation Spectroscopy, Biophys. J., 84 (2003), pp.1960-1968.

[70] S. Boeckle,K. von Gersdorff, S. van der Piepen, C. Culmsee, E. Wagner, and M.Ogris, Purification of polyethylenimine polyplexes highlights the role of free polycations in gene transfer, J. Gene Medicine, 6 (2004), pp.1102-1111.

[71] Arhel N. et al Quantitative four-dimensional tracking of cytoplasmic and nuclear HIV-1 complexes, Nat. Methods, 3 (2006), pp.811-824.

[72] M. Lakadamyali, M.J. Rust, and Z. Zhuang, Endocytosis of influenza viruses, Micro. Infection, 6 (2004), pp.929-936. 
[73] P.S. Rachakonda et al, The relevance of salt bridges for the stability of the influenza virus hemagglutinin, FASEB J., 21 (2007), pp.995-1002.

[74] T. Lagache, C. Sieben, A. Herrmann, and D. Holcman Submitted.

[75] M. Krumbiegel, A. Herrmann, and R. Blumenthal, Kinetics of the low pH-induced conformational changes and fusogenic activity of influenza hemagglutinin, Biophys. J., 67 (1994),pp.2355-2360.

[76] T. Sakai et al, Dual wavelength imaging allows analysis of membrane fusion of influenza virus inside cells, J. Virol., 80 (2006),pp.2013-2018.

[77] M. Lakadamyali et al, Visualizing infection of individual influenza viruses, Proc. Natl. Acad. Sci. U S A, 100 (2003),pp.9280-9285.

[78] C. Knessl , M. Mangel, B.J. Matkowsky, and Z. Schuss, Solution of Kramers-Moyal equations for problems in chemical physics, J. Chem. Phys., 81 (1984), pp.1285-1293.

[79] B. Matkowsky , Z. Schuss, C. Knessl, C. Tier, and M. Mangel, Asymptotic solution of the Kramers-Moyal equation and first-passage times for Markov jump processes, Phys. Rev. A, 29 (1984), pp.3359-3369.

[80] C. Knessl, B.J. Matkowsky, Z. Schuss, and C. Tier, An asymptotic theory of large deviations for Markov jump processes, SIAM J. Appl. Math., 45 (1984), pp. 1006-1102.

[81] Z. Schuss, Theory and Applications of Stochastic Processes, Springer, 2010.

[82] N.G. Van Kampen, Stochastic Processes in Physics and Chemistry. North-Holland, Amsterdam, Netherlands, 1992.

[83] Q. Huang, R. Opitz, E. W. Knapp, and A. Herrmann, Protonation and stability of the globular domain of influenza virus hemagglutinin, Biophys. J., 82 (2002), pp.1050-1058.

[84] Z. Schuss, A. Singer, and D. Holcman, The narrow escape problem for diffusion in cellular microdomains, Proc. Natl. Acad. Sci. U S A, 104 (2007), pp.16098-16103.

[85] L. Pelkmans, T. Birli, M. Zerial, and A. Helenius, Caveolin-stabilized membrane domains as multifunctional transport and sorting devices in endocytic membrane traffic, Cell,118(2004),PP.767-780.

[86] E. Leikina et al, Influenza hemagglutinins outside of the contact zone are necessary for fusion pore expansion, J. Biol. Chem., 279 (2004),pp.26526-26532.

[87] M. Imai, T. Mizuno, and K. Kawasaki, Membrane fusion by single influenza hemagglutinin trimers. Kinetic evidence from image analysis of hemagglutinin-reconstituted vesicles, J. Biol. Chem., 281 (2006),pp.12729-12735.

[88] M. Lakadamyali, M. J. Rust, and X. Zhuang, Ligands for clathrin-mediated endocytosis are differentially sorted into distinct populations of early endosomes, Cell, 124 (2006),pp. 997-1009. 
[89] T.K. Korte et al, Conformational intermediates and fusion activity of influenza virus hemagglutinin, J. Virol., 73 (1999), pp.4567-4574.

[90] R. Fritz, K. Stiasny, F.X. Heinz, Identification of specific histidines as pH sensors in flavivirus membrane fusion, J. Cell Biol., 183 (2008), pp.353-361.

[91] S. Roche, F.A. Rey, Y. Gaudin, and S. Bressanelli, Structure of the prefusion form of the vesicular stomatitis virus glycoprotein $G$, Science, 315 (2007), pp.843-848.

[92] K. Chandran et al, Endosomal proteolysis of the Ebola virus glycoprotein is necessary for infection, Science, 308 (2005), pp.1643-1645.

[93] S. Belouzard, V.C. Chu, and G.R. Whittaker, Activation of the SARS coronavirus spike protein via sequential proteolytic cleavage at two distinct sites, Proc. Natl. Acad. Sci. U S A, 106 (2009), pp.5871-5876.

[94] M. Dominska, and D.M. Dykxhoorn, Breaking down the barriers: siRNA delivery and endosome escape, J. Cell Sci., 123 (2010) pp.1183-1189.

[95] G. Seisenberger et al, Real-time single-molecule imaging of the infection pathway of an adeno-associated virus, Science, 294 (2001) , pp.1929-1932.

[96] B. Brandenburg, and X. Zhuang, Virus trafficking: learning from single-virus tracking, Nat. Rev. Microbiology, 5 (2007), pp.197-208.

[97] O. Seksek, J. Biwersi, and A.S. Verkman, Translational diffusion of macromolecule-sized solutes in cytoplasm and nucleus, J. Cell Biol.,138 (1997), pp.131-142.

[98] HC. Berg, Random walks in Biology. Princeton University Press, 1983.

[99] Risken H: The Fokker-Planck Equation: Methods of Solutions and Applications, Springer, 1996.

[100] A. Singer, Z. Schuss, A. Osipov, D. Holcman, Partially reflected diffusion, SIAM. J. Appl. Math., 68 (2007), pp. 844-868.

[101] T. Lagache, D. Holcman, Effective motion of a virus trafficking inside a biological cell, SIAM. J. Appl. Math., 68 (2008), pp. 1146-1167.

[102] T. Lagache, D. Holcman, Quantifying intermittent transport in cell cytoplasm Phys. Rev. E, 77 (2008) article 030901.

[103] D. Holcman Modeling viral and DNA trafficking in the cytoplasm of a cell, J. Stat. Phys., 127 (2007) pp. 471-494.

[104] T. Lagache, E. Dauty, D. Holcman, Quantitative analysis of virus and plasmid trafficking in cells, Phys. Rev. E,. 79 (2009) article 011921.

[105] Maul GG, L. Deaven, Quantitative determination of nuclear pore complexes in cycling cells with differing DNA content, J. Cell. Biol., 73 (1977) pp. 748-760. 
[106] D. Holcman and Z. Schuss, Diffusion escape through a cluster of small absorbing windows, J. Phys. A: Math. Theor., 41 (2008), article 155001.

[107] D. Coombs, R. Straube and M.J. Ward, Diffusion on a Sphere with Localized Traps: Mean First Passage Time, Eigenvalue Asymptotics, and Fekete Points, SIAM J. Appl. Math., 70 (2009) pp. 302-332.

[108] J. Reingruber, E. Abad and D. Holcman, Narrow escape time to a structured target located at the boundary of a microdomian, J. Chem. Phys., 130 (2009) pp. 094909094916.

[109] HC. Berg, E.M. Purcell Physics of chemoreception, Biophys J., 20 (1977) pp. 193-219.

[110] Lagache T., D. Holcman Submitted.

[111] Y. Han et al., Cultivation of Recombinant Chinese hamster ovary cells grown as suspended aggregates in stirred vessels, J. Biosci. Bioeng., 102 (2006) pp.430-435. 